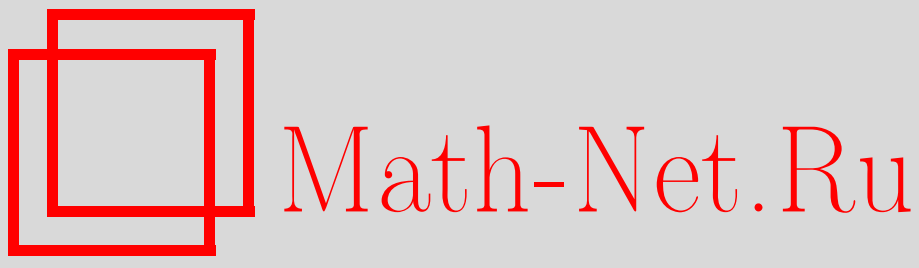

С. А. Назаров, М. В. Олюшин, Приближение гладких контуров многоугольными. Парадоксы в задачах для системы Ламе, Изв. РАН. Сер. матем., 1997, том 61, выпуск 3, 159-186

DOI: https://doi.org/10.4213/im129

Использование Общероссийского математического портала Math-Net.Ru подразумевает, что вы прочитали и согласны с пользовательским соглашением http://www . mathnet.ru/rus/agreement

Параметры загрузки:

IP : 54.164 .48 .24

26 апреля 2023 г., 17:56:26 
УДК 517.946

\author{
С. А. Назаров, М. В. Олюшин
}

\title{
Приближение гладких контуров многоугольными. Парадоксы в задачах для системы Ламе
}

\begin{abstract}
При различных краевых условиях исследуется сходимость решений краевых задач для системы Ламе при аппроксимации гладкого контура многоугольным. Выясняется, в каких случаях возникает парадокс, аналогичный парадоксу Сапонджяна-Бабушки. Приводится формальный асимптотический анализ, содержащий построение пограничных слоев около быстро осциллирующей границы и асимптотических поправок вблизи угловых точек. Приводится обоснование построенной асимптотики.

Библиография: 39 наименований.
\end{abstract}

\section{§1. Постановка задачи, описание и обсуждение результатов}

1. Область и основная краевая задача. Известно (см. [1], [2]), что продольная деформация тонкой, однородной, трехмерной пластины описывается решением двумерной задачи об обобщенном плоском напряженном состоянии. Далее при различных краевых условиях исследуется сходимость решений таких краевых задач при аппроксимации гладкого контура многоугольным и выясняется, при каких условиях возникает парадокс Сапонджяна-Бабушки (см. [3], [4] для случая изгиба пластины).

Сначала сформулируем упомянутую задачу в области с гладкой границей; при этом назначим те краевые условия, которым в работе уделяется наибольшее внимание, и назовем полученную задачу основной.

Пусть $\Omega$ - двумерная область на плоскости $\mathbb{R}^{2}$, ограниченная гладкими, простыми, замкнутыми, выпуклыми контурами $\Gamma_{0}$ и $\Gamma_{\mathrm{D}}$. Не ограничивая общности, длину контура $\Gamma_{0}$ считаем единичной. Вектор смещений $u=\left(u_{1}, u_{2}\right)$ удовлетворяет системе Ламе

$$
L\left(\nabla_{x}\right) u(x) \equiv-\mu \nabla_{x} \cdot \nabla_{x} u(x)-(\lambda+\mu) \nabla_{x} \nabla_{x} \cdot u(x)=f(x), \quad x \in \Omega,
$$

где $x=\left(x_{1}, x_{2}\right)$ - декартовы координаты, $\nabla_{x}=\operatorname{grad}, \mu>0, \lambda \geqslant 0$-коэффициенты Ламе, точкой обозначено скалярное произведение, а $f=\left(f_{1}, f_{2}\right)$-вектор массовых сил.

Декартовы компоненты тензоров деформаций $\varepsilon(u)$ и напряжений $\sigma(u)$ определяются формулами

$$
\varepsilon_{i j}(u)=\frac{1}{2}\left(\frac{\partial u_{i}}{\partial x_{j}}+\frac{\partial u_{j}}{\partial x_{i}}\right), \quad \sigma_{i j}(u)=2 \mu \varepsilon_{i j}(u)+\delta_{i, j} \lambda\left(\varepsilon_{11}(u)+\varepsilon_{22}(u)\right) .
$$

Здесь $i, j=1,2$, а $\delta_{i, j}$ - символ Кронекера. В окрестности $\mathscr{U}$ контура $\Gamma_{0}$ введем естественные координаты $(n, s)$, где $s \in[0,1)$ - длина дуги вдоль $\Gamma_{0}$, а $n$ - расстояние 
до $\Gamma_{0}$ по нормали, причем $n>0$ в $\mathscr{U} \cap \Omega$. Пусть еше $\mathbf{n}=\left(n_{1}, n_{2}\right)$ и $\mathbf{s}=\left(n_{2},-n_{1}\right)-$ нормальный и касательньй векторы к границе $\Gamma_{0}$.

Назначим следующие краевые условия:

$$
\begin{gathered}
u(x)=0, \quad x \in \Gamma_{\mathrm{D}} \\
u_{n}(x)=0, \quad \sigma_{n s}(u ; x)=0, \quad x \in \Gamma_{0} .
\end{gathered}
$$

В (1.3) $u_{n}$ и $\sigma_{n s}-$ компоненты вектора смешений и тензора напряжений в координатах $(n, s)$,

$$
\begin{gathered}
u_{n}=\mathbf{n} \cdot u, \quad u_{s}=\mathbf{s} \cdot u, \\
\sigma_{n s}(u)=\mathbf{n} \cdot \sigma(u) \mathbf{s}, \quad \sigma_{n n}(u)=\mathbf{n} \cdot \sigma(u) \mathbf{n} .
\end{gathered}
$$

Условие (1.2) означает жесткое защемление контура $\Gamma_{\mathrm{D}}$, a (1.3) - условие идеального (без трения и отрыва) контакта боковой поверхности пластины с абсолютно жестким профилем $\Gamma_{0}$.

Известно (см., например, [5], [6]), что при $f \in L_{2}(\Omega)$ сушествует единственное обобшенное решение $u \in W_{2}^{1}(\Omega)$ задачи (1.1)-(1.3). Если $f \in C^{\infty}(\bar{\Omega})$, то такое решение оказывается гладким в $\bar{\Omega}$.

Опишем аппроксимацию контура $\Gamma_{0}$. Пусть $N$ - большое натуральное число и $P^{1}, \ldots, P^{N-1}, P^{N} \equiv P^{0}$ - точки на контуре $\Gamma_{0}$, разбивающие его на $N$ дуг с длинами $\varepsilon=N^{-1}$. Соединим точки $P^{k}$ и $P^{k+1}$ прямолинейньги отрезками и обозначим $\Gamma_{N}$ построенную ломаную. В области $\Omega_{N}$, ограниченной контурами $\Gamma_{N}$ и $\Gamma_{\mathrm{D}}$, рассмотрим краевую задачу

$$
\begin{gathered}
L\left(\nabla_{x}\right) v^{N}(x)=f(x), \quad x \in \Omega_{N}, \\
v^{N}(x)=0, \quad x \in \Gamma_{\mathrm{D}}, \\
v_{\nu}^{N}(x)=0, \quad \sigma_{\nu \tau}\left(v^{N} ; x\right)=0, \quad x \in \Gamma_{N} \backslash \mathscr{P} .
\end{gathered}
$$

Здесь $\mathscr{P} \equiv\left\{P^{1}, \ldots, P^{N}\right\}, \nu$ и $\tau$ - нормальная и касательная координаты, отнесенные к звеньям ломаной $\Gamma_{N}$, а $v_{\nu}^{N}$ и $\sigma_{\nu \tau}\left(v^{N}\right)$ определены аналогично (1.4).

Одной из целей работы является построение асимптотики решения задачи (1.5)-(1.7) при $N \rightarrow \infty(\varepsilon \rightarrow 0)$.

2. Обсуждение. В [7] (см. также книгу [3]) было обнаружено значительное расхождение в результатах решений задач об изгибе круглой пластины со свободно опертым краем и аппроксимирующей ее многоугольной пластины при тех же краевых условиях на $N$ сторонах многоугольника, вписанного в круг. В [4] этот факт нашел теоретическое обоснование: оказывается, решение задачи о многоугольной пластине при $N \rightarrow \infty$ сходится к решению задачи в круге с краевыми условиями, отличаюшимися от условия свободного опирания. В [8] установлено, что эти предельные условия зависят от знака кривизны гладкого контура - в случае многоугольного отверстия ответ получился иным.

Поскольку устойчивость решения по отношению к малой вариации границы считается необходимым свойством математической модели, то обнаруженные в [3], $[4],[7],[8]$ факты рассматриваются как парадоксальные. Их механическому истолкованию посвящено большое число исследований (см. [9]-[14] и др.). По мнению авторов, одну из причин следует искать в асимптотической природе двумерных уравнений теории пластин - они получаются (см. [1], [2], [6] и др.) в результате 
асимптотического интегрирования трехмерных уравнений теории упругости при интерпретации относительной толщины $h$ пластины как малого параметра. Наличие двух параметров, $N$ и $h$, требует аккуратного обращения с предельными переходами (при $N^{-1} \ll h$ правомочность использования уравнений и краевых условий двумерной теории пластин Кирхгофа вызьвает сомнения).

При изучении трехмерной задачи об изгибе пластины (многоугольной призме $\Omega_{N} \times(-h / 2, h / 2)$ малой высоты $\left.h\right)$ в [15] установлено, что появление парадокса зависит от того, какие краевые условия выставляются на боковой поверхности. Именно, условия

$$
u_{3}(x)=0, \quad \sigma_{\nu \tau}(u ; x)=0, \quad \sigma_{\nu \nu}(u ; x)=0, \quad x \in \Gamma_{N} \times(-h / 2, h / 2),
$$

допускают предельный переход, а условия

$$
u_{3}(x)=0, \quad u_{\tau}(x)=0, \quad \sigma_{\nu \nu}(u ; x)=0, \quad x \in \Gamma_{N} \times(-h / 2, h / 2),
$$

его не допускают (здесь вектор смещений и тензор напряжений трехмерные, ось $0 x_{3}$ перпендикулярна основанию пластины, а остальные обозначения - те же, что и в п. 1). Соотношения (1.8) назьваются условиями свободного опирания (simple support), a (1.9) - условиями жсесткого опирания (hard support). Важным моментом оказывается то, что в двумерной теории пластин Кирхгофа краевые условия (1.8) и (1.9) не различаются - обе группы условий в результате асимптотического интегрирования (при $h \rightarrow 0$ ) порождают классические условия свободного опирания.

Другой вывод работы [15] представляется авторам спорным. В [15] отмечается, что в задаче для системы уравнений пластин Рейснера [16], [17] равенства (1.8) и (1.9) порождают различные краевые условия, которые наследуют возможность предельных переходов при $N \rightarrow \infty$ от трехмерной задачи. На основании этого утверждается, что модель Рейснера является более точной, чем модель Кирхгофа. В то же время известно (см. [18], [19] и др.), что асимптотические погрешности теорий пластин Кирхгофа и Рейснера одинаковы по порядку малого параметра $h$. Вероятнее всего, упомянутое совпадение возможностей предельных переходов случайно или вызвано иными причинами не асимптотического характера.

3. Предварительное описание результатов. Для изотропных пластин состояние изгиба и плоской деформации разделяются, т.е. находятся при решении несвязанных задач (бигармоническое уравнение и двумерная система Ламе). Предельные переходы в задачах для системы Ламе не изучались - настоящая статья восполняет этот пробел. Рассматриваются четыре типа краевых условий. Как уже говорилось, основное внимание уделяется краевым условиям (1.7) - на сторонах многоугольника фиксируются нормальное смешение и касательное напряжение. В этом случае возникает парадокс, аналогичный парадоксу Сапонджяна-Бабушки [3], [4]: предел $v$ решений $v^{\prime}$ задач (1.5)-(1.7) удовлетворяет уравне- 
ниям

$$
\begin{gathered}
L\left(\nabla_{x}\right) v(x)=f(x), \quad x \in \Omega_{N}, \\
v(x)=0, \quad x \in \Gamma_{\mathrm{D}}, \\
v_{n}(x)=0, \quad x \in \Gamma_{0}, \\
\sigma_{n s}(v ; x)= \pm \frac{1}{2} v_{s}(x) \mathbb{k}(s)\left[\lambda\left(1-\frac{1}{\varkappa}\right)(1 \mp 1)+2 \mu\right. \\
\left.+\mu\left(1-\frac{1}{\varkappa} \pm\left(1+\frac{1}{\varkappa}\right)\right)\right], \quad x \in \Gamma_{0}
\end{gathered}
$$

$(\varkappa=(\lambda+3 \mu) /(\lambda+\mu))$, причем, как и в [8], предельные условия (1.13) различаются в случаях внешнего $(+)$ или внутреннего $(-)$ положения контура $\Gamma_{0}$. Формальньй асимптотический анализ, содержащий построение пограничных слоев около быстро осциллирующей гранищы $\Gamma_{N}$ и асимптотических поправок в непосредственной близости от угловых точек $P^{k}$, приведен в $\S 2$. Обоснованию асимптотики посвяшен $\S 3$ (в частности, там доказывается независимость от $N$ константы в неравенстве Корна для области $\left.\Omega_{N}\right)$.

В $\S 4$ кратко обсуждаются оставшиеся три типа краевых условий: Дирихле (жестко защемленный край), Неймана (свободный край) и смешанные условия (на $\Gamma_{N}$ фиксируются касательные смешения и нормальные напряжения). Как и следовало ожидать, в первых двух случаях после предельных переходов возникают соответственно условия Дирихле и Неймана на $\Gamma_{0}$. Третьему случаю присуши те же аномалии, что и при условии (1.7) - в качестве предельных выступают условия типа упругой заделки.

Отметим напоследок, что соотношениям (1.8) в трехмерной задаче отвечают условия Неймана в задаче о продольной деформации пластины $\Omega_{N}$, а условиям (1.9) условия

$$
v_{\tau}^{N}(x)=0, \quad \sigma_{\nu \nu}\left(v^{N} ; x\right)=0, \quad x \in \Gamma_{N} .
$$

Асимптотические свойства решений этих задач, обсуждаемые в $\S 4$, показывают, что и для плоского напряженного состояния пластины характерны эффекты, обнаруженные в [15] для пластин Рейснера. Такая аналогия побуждает взять условия (1.14) в качестве основных и уделить им наибольшее внимание, однако механическая интерпретация условий (1.7) ясна (контакт без трения с абсолютно жестким профилем), а как реализовать практически условия (1.14), авторы не знают.

\section{§2. Формальная асимптотика}

1. Пограничный слой около $\Gamma_{0}$. В качестве главного члена асимптотики решения $v^{N}$ задачи (1.5)-(1.7) возьмем гладкую в $\bar{\Omega}$ функцию $v$, удовлетворяюшую системе уравнений (1.1) и краевым условиям (1.2) на $\Gamma_{\mathrm{D}}$. Краевые условия на $\Gamma_{\mathrm{D}}$ можно найти только после построения пограничного слоя около осциллирующей гранищы $\Gamma_{N}$ (см. [20]-[24] и др.). Этот пограничный слой описывается при помощи быстрых переменных

$$
\xi=\left(\xi_{1}, \xi_{2}\right), \quad \xi_{1}=\varepsilon^{-1} s, \quad \xi_{2}=\varepsilon^{-1} n,
$$

и имеет вид

$$
\varepsilon w(s, \xi)+\cdots,
$$


где $w=\left(w_{s}, w_{n}\right)$, а функции $w_{n}, w_{s}$, зависяшие гладко от переменной, являются периодическими по $\xi_{1}$ (с единичным периодом) и экспоненциально затухают при $\xi_{2} \rightarrow \infty$. Будем считать, что длина дуги $s$ отмеряется от точки $P^{0}$. Если формально положить $\varepsilon=0 \quad(N=\infty)$ и учесть периодическую структуру границы $\Gamma_{N}$, то областью изменения переменной $\xi$ естественно назначить полуполосу $\Pi=(-1 / 2,1 / 2) \times(0,+\infty)$, причем на ее боковых сторонах $\pi_{ \pm}$нужно поставить условие периодичности

$$
\frac{\partial^{k} w}{\partial \xi_{1}^{k}}\left(s,-\frac{1}{2}, \xi_{2}\right)=\frac{\partial^{k} w}{\partial \xi_{1}^{k}}\left(s,+\frac{1}{2}, \xi_{2}\right), \quad \xi_{2}>0, \quad k=0,1 .
$$

Найдем систему уравнений для вектор-функции $w$. С этой целью запишем в $\Omega \cap U$ однородную (правая часть $f$ компенсирована решением $v$ ) систему $(1.1)$ при помощи координат $(s, n)$ :

$$
\begin{aligned}
& \mu\left\{\partial_{n}^{2} u_{n}+\left(\partial_{n} u_{n}\right) K(n, s) \partial_{n} K^{-1}(n, s)+K(n, s) \partial_{s}\left(K(n, s) \partial_{s} u_{n}\right)\right. \\
& \quad+\partial_{s}\left(K^{-1}(n, s) u_{s}\right) \partial_{n} K^{2}(n, s)+u_{n} \partial_{n}\left(K(n, s) \partial_{n} K^{-1}(n, s)\right) \\
& \left.\quad+K^{-1}(n, s) u_{s} \partial_{n}\left(K(n, s) \partial_{s} K(n, s)\right)\right\} \\
& \quad+(\lambda+\mu) \partial_{n}\left\{K(n, s) \partial_{n}\left(K^{-1}(n, s) u_{n}\right)+K(n, s) \partial_{s} u_{s}\right\}=0, \\
& \mu\left\{\partial_{n}\left(K(n, s) \partial_{n}\left(K^{-1}(n, s) u_{s}\right)\right)+\partial_{s}\left(K^{3}(n, s) \partial_{s}\left(K^{-1}(n, s) u_{s}\right)\right)\right. \\
& \quad+K(n, s) u_{n} \partial_{s}\left(K(n, s) \partial_{n} K^{-1}(n, s)\right)-K^{-1}(n, s)\left(\partial_{s} u_{n}\right) \partial_{n} K^{2}(n, s) \\
& \left.\quad+u_{s} \partial_{s}\left(K(n, s) \partial_{s} K(n, s)\right)\right\} \\
& +(\lambda+\mu) K(n, s) \partial_{s}\left\{K(n, s) \partial_{n}\left(K^{-1}(n, s) u_{n}\right)+K(n, s) \partial_{s} u_{s}\right\}=0 .
\end{aligned}
$$

Здесь $K(n, s)=(1+n \mathbb{k}(s))^{-1}, \mathbb{k}(s)$ - кривизна (положительная в том случае, когда $\Gamma_{0}-$ внешний контур, и отрицательная, если он внутренний), $\partial_{s}=\partial / \partial s$, $\partial_{n}=\partial / \partial n, v_{n}$ и $v_{s}$ определены в (1.4).

Переходя к быстрым переменным $(2.2)$, выделяем главную часть $\varepsilon^{-2} L\left(\nabla_{\xi}\right)$ оператора системы (2.4). Таким образом, главньй член пограничного слоя обязан удовлетворять уравнению

$$
-L\left(\nabla_{\xi}\right) w(s, \xi)=0, \quad \xi \in \Pi
$$

Подчеркнем, что здесь компонента $w_{s}$ объявляется первой, а $w_{n}$ - второй; может быть, несколько вольно, пишем $w_{1}=w_{s}, w_{2}=w_{n}$, т.е. интерпретируем криволинейные координаты $(s, n)$ как декартовы.

Осталось найти краевое условие для $w$ на торце $\pi_{0}=\left\{\xi: \xi_{2}=0,\left|\xi_{1}\right|<\right.$ $1 / 2\}$ полуполосы. Прежде всего отметим, что краевое условие (1.12) очевидно (см. также пояснение в замечании 2.1). Поэтому, раскладывая функции в ряд Тейлора по переменной $n$, получаем

$$
\begin{gathered}
v_{n}(s, n)=n V_{n}^{1}(s)+O\left(n^{2}\right), \\
v_{s}(s, n)=V_{s}^{0}(s)+n V_{s}^{1}(s)+O\left(n^{2}\right), \\
\sigma_{n s}(v ; s, n)=\Sigma_{n s}^{0}(s)+O(n) .
\end{gathered}
$$


Вычислим теперь главные части невязок, оставляемые $v$ в краевых условиях (1.7).

Каждая из вершин ломаной $\Gamma_{N}$ попадает в центр $\xi=0$ торца $\pi_{0}$. Выберем одну из вершин, для определенности $P^{0}$. Уравнения двух звеньев $\gamma^{ \pm}$ломаной, исходящих из $P^{0}$, имеют вид

$$
n=\frac{1}{\mathbb{k}}-\frac{1}{\mathbb{k}} \frac{\cos [\mathbb{k} /(2 N)]}{\cos [\mathbb{k}(|s|-1 /(2 N))]}, \quad \mathbb{k}=\mathbb{k}(0) .
$$

В координатах $\left(\xi_{1}, \xi_{2}\right)$ уравнение гранищы $\gamma^{ \pm}$записьвается следуюшим образом:

$$
\xi_{2}=-\varepsilon \mathbb{k} \frac{1}{2}\left[\left(\left|\xi_{1}\right|-\frac{1}{2}\right)^{2}-\frac{1}{4}\right]+O\left(\varepsilon^{2}\right) .
$$

Кроме того, компоненты $\nu_{n}^{ \pm}$и $\nu_{s}^{ \pm}$вектора нормали к $\gamma^{ \pm}$удовлетворяют соотношениям

$$
\begin{aligned}
& \nu_{n}^{ \pm}(\xi)=1+O\left(\varepsilon^{2}\right), \\
& \nu_{s}^{ \pm}(\xi)=\varepsilon \mathbb{k}\left(\xi_{1} \mp 1 / 2\right)+O\left(\varepsilon^{2}\right) .
\end{aligned}
$$

Согласно первым двум формулам в (2.6), а также представлениям (2.7) и (2.8) получаем, что на $\gamma^{ \pm}$

$$
\begin{aligned}
v_{\nu^{ \pm}}(s, n)= & v_{n}(s, n) \nu_{n}^{ \pm}(\xi)+v_{s}(s, n) \nu_{s}^{ \pm}(\xi)=\left[\varepsilon \xi_{2} V_{n}^{1}+O\left(\varepsilon^{2} \xi_{2}^{2}\right)\right]\left[1+O\left(\varepsilon^{2}\right)\right] \\
& +\left[V_{s}^{0}+O\left(\varepsilon \xi_{2}\right)\right]\left[\varepsilon \mathbb{k}\left(\xi_{1} \mp 1 / 2\right)+O\left(\varepsilon^{2}\right)\right] \\
= & \varepsilon \mathbb{k}\left\{V_{s}^{0}\left(\xi_{1} \mp \frac{1}{2}\right)-\frac{1}{2}\left[\left(\left|\xi_{1}\right|-\frac{1}{2}\right)^{2}-\frac{1}{4}\right] V_{n}^{1}\right\}+O\left(\varepsilon^{2}\right) .
\end{aligned}
$$

Кроме того, в силу $(2.6),(2.8)$ и (1.4)

$$
\sigma_{\nu \tau}(v ; x)=\Sigma_{n s}^{0}+O(\varepsilon)
$$

Пограничный слой $\varepsilon w$ из $(2.2)$ должен компенсировать члены $O(\varepsilon)$ и $O(1)$ соответственно в $(2.9)$ и в $(2.10)$, т.е. краевые условия на торце $\pi_{0}$ полуполосы выглядят так:

$$
\begin{gathered}
w_{2}\left(s, \xi_{1}, 0\right)=- \\
-\mathbb{k}(s)\left\{V_{s}^{0}(s)\left(\xi_{1}-\frac{1}{2} \operatorname{sgn}\left(\xi_{1}\right)\right)\right. \\
\left.-\frac{1}{2} V_{n}^{1}(s)\left[\left(\left|\xi_{1}\right|-\frac{1}{2}\right)^{2}-\frac{1}{4}\right]\right\}, \\
\mu\left(\frac{\partial w_{2}}{\partial \xi_{1}}\left(s, \xi_{1}, 0\right)+\frac{\partial w_{1}}{\partial \xi_{2}}\left(s, \xi_{1}, 0\right)\right)=-\Sigma_{n s}^{0}(s), \quad\left|\xi_{1}\right|<\frac{1}{2} .
\end{gathered}
$$

Заметим, что правая часть условия (2.11) обладает разрывом первого рода в точке $\xi_{1}=0$. Задача $(2.5),(2.3),(2.11),(2.12)$ имеет ограниченное решение, однако из-за особенностей $O\left(|\xi|^{-1}\right)$ производных в точке $\xi=0$ это решение не попадает в $W_{2, \text { loc }}^{1}(\Pi)$. Более того, как будет показано далее, в формировании пограничного слоя участвуют иррегулярные решения

$$
w(s, \xi)=O(\ln |\xi|), \quad|\xi| \rightarrow 0 .
$$

Условие (2.13) уже не гарантирует единственность решения. С целью его уточнения в следующем разделе изучается поведение $v^{N}$ вблизи угловых точек границы $\Gamma_{N}$. 
2. Специальное решение в угловой области. Пусть $(r, \varphi)$ - полярные координаты на плоскости, $\xi_{1}=-r \sin \varphi, \xi_{2}=r \cos \varphi$ и $K=\{x: r>0,|\varphi|<\alpha\}-$ угол с вершиной 0 ; считаем, что его полураствор $\alpha$ мало отличается от $\pi / 2$. Далее рассматриваются случаи $\alpha=\pi / 2$ (полуплоскость) и

$$
\alpha=\mp \delta+\pi / 2 \text {; }
$$

здесь $\delta$ - мальй положительный параметр. Согласно (2.8) полураствор $\alpha_{j}$ угла с вершиной $P^{j} \in \Gamma_{N}$ удовлетворяет соотношению

$$
\alpha_{j}=\frac{\pi}{2}-\varepsilon \mathbb{k}\left(P^{j}\right)+O\left(\varepsilon^{2}\right) .
$$

При этом если $\Gamma_{N}-$ внешний контур, то $\mathbb{k}\left(P^{j}\right)>0$ и, значит, в (2.14) берется знак “-". В случае внутреннего контура $\Gamma_{N}$ в $(2.14)$ выбирается “+”, так как $\mathbb{k}\left(P^{j}\right)<0$.

Подчеркнем, что на протяжении всего параграфа приходится различать эти два случая - им отвечают разные предельные краевые условия на $\Gamma_{0}$.

В соответствии с общими результатами [25] (см. также [26], [27]) для описания поведения $v^{N}$ около $P^{j}$ необходимо перечислить степенные решения

$$
r^{\Lambda} \Phi(\varphi, \ln r)
$$

однородной системы Ламе в $K$ с краевыми условиями $(1.3)$ на $\partial K($ в $(2.16) \Phi-$ полином переменной $\ln r$; cp. с (2.21)). Нас интересуют главные члены разложений $v^{N}$, и потому мы указываем только такие решения, которые имеют мальй показатель $\Lambda$ :

$$
r^{-1+\pi /(2 \alpha)} \Phi^{+}(\varphi), \quad r^{1-\pi /(2 \alpha)} \Phi^{-}(\varphi) .
$$

Угловые части $\Phi^{ \pm}$задаются формулами

$$
\begin{aligned}
\Phi_{r}^{+}(\varphi) & =\sin \frac{\pi \varphi}{2 \alpha}, & \Phi_{\varphi}^{+}(\varphi) & =\cos \frac{\pi \varphi}{2 \alpha} ; \\
\Phi_{r}^{-}(\varphi) & =\left(\varkappa-1+\frac{\pi}{2 \alpha}\right) \sin \frac{\pi \varphi}{2 \alpha}, & \Phi_{\varphi}^{-}(\varphi) & =\left(\varkappa+1-\frac{\pi}{2 \alpha}\right) \cos \frac{\pi \varphi}{2 \alpha}
\end{aligned}
$$

(cp. с $[28, \S 3.8])$. Здесь $\varkappa=(\lambda+3 \mu)(\lambda+\mu)^{-1}, \Phi_{r}$ и $\Phi_{\varphi}$ - полярные компоненты,

$$
\begin{array}{ll}
\Phi_{1}=\Phi_{r} \sin \varphi+\Phi_{\varphi} \cos \varphi, & \Phi_{2}=\Phi_{r} \cos \varphi-\Phi_{\varphi} \sin \varphi ; \\
\Phi_{r}=\Phi_{1} \sin \varphi+\Phi_{2} \cos \varphi, & \Phi_{\varphi}=\Phi_{1} \cos \varphi-\Phi_{2} \sin \varphi .
\end{array}
$$

Поскольку решение $v^{N}$ принадлежит $W_{2}^{1}\left(\Omega_{N}\right)$, то его разложение может содержать только тот из векторов (2.17), у которого показатель степени положителен. Таким образом, в случае $\alpha<\pi / 2$ выбираем $r^{-1+\pi /(2 \alpha)} \Phi^{+}(\varphi)$, а в случае $\alpha>\pi / 2$ выбираем $r^{1-\pi /(2 \alpha)} \Phi^{-}(\varphi)$.

Наконец, для $\alpha=\pi / 2$ имеются такие решения:

$$
r^{0} \Phi^{0}(\varphi), \quad r^{0} \Phi^{1}(\varphi, \ln r) ;
$$


здесь

$$
\begin{aligned}
\Phi_{r}^{0}(\varphi)=\sin \varphi, & \Phi_{\varphi}^{0}(\varphi)=\cos \varphi \\
\Phi_{r}^{1}(\varphi, \ln r)=\left[\ln r-(2 \varkappa)^{-1}\right] \sin \varphi, & \Phi_{\varphi}^{1}(\varphi, \ln r)=\left[\ln r+(2 \varkappa)^{-1}\right] \cos \varphi .
\end{aligned}
$$

Подчеркнем, что первый вектор в (2.18) является жестким поступательным смещением вдоль гранищы полуплоскости, а второй отвечает силе, сосредоточенной в начале координат и направленной вдоль луча $\varphi=\pi / 2$.

В соответствии с (2.14) величины (2.17) зависят от малого параметра $\delta$. Применим формулу Тейлора (по переменной $\delta$ ):

$$
\begin{aligned}
r^{\mp 1 \pm \pi /(2 \alpha)} \Phi^{ \pm}(\varphi) & =\exp [\mp(1-\pi /(2 \alpha)) \ln r] \Phi^{ \pm}(\varphi) \\
& =\Phi^{0}(\varphi)+2 \delta \pi^{-1} \Phi^{1}(\varphi, \ln r) \mp 2 \delta \pi^{-1} \Psi(\varphi)+O\left(\delta^{2}|\ln r|^{2}\right) .
\end{aligned}
$$

При этом

$$
\Psi_{r}(\varphi)=-\varphi \cos \varphi-(2 \varkappa)^{-1} \sin \varphi, \quad \Psi_{\varphi}(\varphi)=\varphi \sin \varphi+(2 \varkappa)^{-1} \cos \varphi .
$$

Первые два слагаемые справа уже возникали в (2.18). Вектор $\Psi$ удовлетворяет системе Ламе в полуплоскости, причем на ее гранище напряжения $\sigma_{21}(\Psi)$ аннулируются и верны условия

$$
\Psi_{2}( \pm \pi / 2)=\mp \Psi_{\varphi}( \pm \pi / 2)=\mp \pi / 2 .
$$

Таким образом, за счет $\Psi$ можно компенсировать скачок краевых условий в (2.11).

3. Асимптотические условия для $w$. Рассуждения в этом пункте формальны (они будут обоснованы в $\S 3$ ), и все остатки в асимптотических формулах обозначаются многоточием.

Обрашаясь к результатам [25] (см. также $[27, \S 6.6]$ ), замечаем, что согласно выкладкам из п. 2 вблизи каждой из вершин $P^{j}$ ломаной $\Gamma_{N}$ для решения $v^{N}$ выполняется представление

$$
v^{N}(x)=K_{j}(\varepsilon) r_{j}^{\mp 1 \pm \pi /\left(2 \alpha_{j}\right)} \Phi^{ \pm}\left(\varphi_{j}\right)+\cdots
$$

В $(2.19)\left(r_{j}, \varphi_{j}\right)$ - полярные координаты с центром $P^{j},\left|\varphi_{j}\right|<\alpha_{j}$, a $K_{j}(\varepsilon)-$ некоторый коэффициент, определяемый данными задачи в целом. Предполагая зависимость $K_{j}$ от $\varepsilon$ гладкой и обращаясь к $(2.19),(2.15)$ и $(2.16)$, вьводим

$$
\begin{aligned}
& v^{N}(x)=K_{j}(0) \Phi^{0}\left(\varphi_{j}\right) \\
& \quad+\varepsilon\left\{\pi^{-1} K_{j}(0) \mathbb{k}\left(P^{j}\right)\left[ \pm \Phi^{1}\left(\varphi_{j}, \ln r_{j}\right)-\Psi\left(\varphi_{j}\right)\right]+\partial_{\varepsilon} K_{j}(0) \Phi^{0}\left(\varphi_{j}\right)\right\}+\cdots
\end{aligned}
$$

Асимптотика решения $v^{N}(x)$ ищется в виде суммы $v(x)+\varepsilon w(s, \xi)$. Согласно $(2.6)$

$$
v(x)+\varepsilon w(s, \xi)=V_{s}^{0}\left(P^{j}\right) \Phi^{0}\left(\varphi_{j}\right)+\varepsilon\left\{w\left(P^{j}, \xi\right)+\xi_{2} V_{s}^{1}(s)\right\}+\cdots
$$

Сравнивая (2.21) с последней формулой, видим, что, во-первых,

$$
K_{j}(0)=V_{s}^{0}\left(P^{j}\right)
$$


и, во-вторых,

$$
w(s, \xi)=\pi^{-1} V_{s}^{0}\left(P^{j}\right) \mathbb{k}\left(P^{j}\right)\left[ \pm \Phi^{1}\left(\varphi_{j}, \ln r_{j}\right)-\Psi\left(\varphi_{j}\right)\right]+\partial_{\varepsilon} K_{j}(0) \Phi^{0}\left(\varphi_{j}\right)+\cdots
$$

Итак, условие (2.13) расшифровывается следующим образом:

$$
\begin{aligned}
w(s, \xi)= & \pi^{-1} V_{s}^{0}(s) \mathbb{k}(s)\left[ \pm \Phi^{1}(\varphi, \ln r)-\Psi(\varphi)\right] \\
& +C(s) \Phi^{0}(\varphi)+O(r|\ln r|), \quad r=|\xi| \rightarrow 0 .
\end{aligned}
$$

Отметим, что слагаемое $\xi_{2} V_{s}^{1}(s)=O(r)$ не принималось во внимание и что связь $C(s)$ с $K_{j}(\varepsilon)$ далее не понадобится. Кроме того, в силу (2.18) член асимптотики, введенный в (2.24), учитывает скачок в краевом условии (2.11). Наконец, вид остатка в (2.24) согласован с полным асимптотическим разложением решения $w$, вытекающим из результатов [25].

4. Условие затухания пограничного слоя. Основным свойством пограничного слоя является его исчезновение на бесконечности с экспоненциальной скоростью. Решение задачи (2.5), (2.3), (2.11), (2.12) обладает этим свойством лишь при дополнительном условии, связывающем коэффициенты разложений (2.6). Именно благодаря этой связи появляется второе предельное краевое условие (1.13)

Теорема 2.1. Решение задачи (2.5), (2.3), (2.11), (2.12), дополненной требованием не более чем степенного роста при $\xi_{2} \rightarrow+\infty$, определено с точностью до слагаемого $c_{1} e^{1}+c_{2} \xi_{2} e^{2}$ (где $e^{i}-$ орт оси $\xi_{i}, c_{i}-$ произвольные константы); оно бесконечно дифференцируемо всюду в $\bar{\Pi}$, за исключением точки $\xi=0$, д допускает разлохсение

$$
w(s, \xi)=c_{1} e^{1}+c_{2} \xi_{2} e^{2}+a_{1} \xi_{2} e^{1}+a_{2} e^{2}+O\left(\exp \left(-\rho \xi_{2}\right)\right), \quad \xi_{2} \rightarrow+\infty,
$$

где $\rho$-некоторое положительное число. Формулу (2.25) можсно дифферениировать с сохранением остатка. Коэффициенты $a_{i}$ определень равенствами

$$
\begin{gathered}
a_{1}=-\frac{1}{\mu} \Sigma_{n s}^{0}(s) \pm \frac{\mathbb{k}(s)}{2 \mu} V_{s}^{0}(s)\left\{\lambda\left(1-\frac{1}{\varkappa}\right)(1 \mp 1)+2 \mu\right. \\
\left.+\mu\left[1-\frac{1}{\varkappa} \pm\left(1+\frac{1}{\varkappa}\right)\right]\right\}, \\
a_{2}=-\frac{\mathbb{k}(s)}{12} V_{n}^{1}(s) .
\end{gathered}
$$

ДокАЗАТЕЛЬСтво. Обратим внимание на два факта. Во-первых, периодическое продолжение правой части $f\left(\xi_{1}\right)$ краевого условия $(2.11)$ является бесконечно дифференцируемым в точках $\xi_{1}= \pm 1 / 2$. Во-вторых, $f$ отличается от функции класса $C^{2}[-1 / 2,1 / 2]$ слагаемым

$$
\frac{1}{2} \mathbb{k}(s)\left\{\operatorname{sgn}\left(\xi_{1}\right) V_{s}^{0}(s)-|\xi| V_{n}^{1}(s)\right\} .
$$

Скачок в точке $\xi=0$ учитывается построенной ранее вектор-функцией $\Psi$. При помощи общей процедуры (см. [25]-[27]) можно найти решение $r \psi(\varphi, \ln r)$, обладающее теми же свойствами, что и $\Psi$, но компенсирующее скачок производной $\partial f / \partial \xi_{1}$, т.е. удовлетворяющее соотношению

$$
r \psi_{2}( \pm \pi / 2, \ln r)=\pi r / 2
$$


вместо (2.20). Подчеркнем, что $\frac{\partial}{\partial \xi_{1}}[r \psi(\varphi, \ln r)]=\Psi(\varphi)$.

Теперь решение $w$ ишем в виде

$$
w(\xi)=w^{0}(\xi)+\chi(r) \pi^{-1} \mathbb{k}(s)\left[V_{s}^{0}(s)\left( \pm \Phi^{1}(\varphi, \ln r)-\Psi(\varphi)\right)-V_{n}^{1}(s) r \psi(\varphi, \ln r)\right]
$$

где $\chi \in C^{\infty}(\mathbb{R})$ - срезаюшая функция, $\chi(t)=0$ при $|t|>1 / 3$ и $\chi(t)=1$ при $|t|<1 / 6$. Заметим, что периодическая вектор-функция $w^{0}$ удовлетворяет соотношениям

$$
\begin{gathered}
L\left(\nabla_{\xi}\right) w^{0}(\xi)=F(\xi), \quad \xi \in \Pi, \\
\mu\left(\frac{\partial w_{1}^{0}}{\partial \xi_{2}}\left(\xi_{1}, 0\right)+\frac{\partial w_{2}^{0}}{\partial \xi_{1}}\left(\xi_{1}, 0\right)\right)=G_{2}\left(\xi_{1}\right), \\
w_{2}^{0}\left(\xi_{1}, 0\right)=G_{1}\left(\xi_{1}\right), \quad \xi \in \pi_{0},
\end{gathered}
$$

в которых $G_{2} \in C^{\infty}[-1 / 2,1 / 2], G_{1} \in C[-1 / 2,1 / 2], F \in C_{0}^{\infty}(\bar{\Pi})$, причем периодическое продолжение сохраняет класс гладкости. Таким образом, можно воспользоваться теоремами 5.1.4 (1,3), 5.4.11 из [27], утверждающими, что задача (2.29) имеет решение в классе функций, растущих на бесконечности не более чем степенным образом. (Ссылку на результаты [27] можно заменить исследованием разрешимости задачи (2.29) в классе вектор-функций с конечным интегралом упругой энергии; ср. с [31], [32].) По теореме о локальном повышении гладкости решений эллиптических задач [31], [32] заключаем, что $w^{0}$ (а значит, и $w$ ) бесконечно дифференцируемо всюду в $\bar{\Pi}$, кроме точки $\xi=0$. Помимо этого, согласно теоремам 5.1.4 (2), 5.4.2 из [27] справедливо асимптотическое представление (2.24) с некоторыми константами $a_{i}, c_{i}$ (нетрудно проверить, что существуют только четыре линейно независимых полиномиальных решения однородной системы Ламе в полосе, подчиненных условиям периодичности (2.3) - все они указаны в (2.25)).

Непосредственно проверяется, что $c_{1} e^{1}$ и $c_{2} \xi_{2} e^{2}$ удовлетворяют однородной задаче $(2.5),(2.3),(2.11),(2.12)$; согласно результатам $[27, \S 5.4]$ линейными комбинациями этих векторов исчерпывается произвол в выборе $w^{0}$ (и $w$ ).

Осталось вычислить множители $a_{i}$ в $(2.25)$. С этой целью воспользуемся методом [26]. Удалим из длинного прямоугольника $\Pi_{t}=(-1 / 2,1 / 2) \times(0, t)$ полукруг с центром $\xi=0$ и малым радиусом $d$. В формулу Грина (тождество Бетти) для полученной области $\Pi_{t, d}$ подставим векторы $w$ и $e^{1}$. Двумерный интеграл исчезнет в силу уравнения (2.5). Интегралы по боковым сторонам сокращаются ввиду условия периодичности. Таким образом, выполняется равенство

$$
\begin{aligned}
0= & I_{1}^{(t)}+I_{2}^{(0)}+I_{3}^{(d)} \equiv \int_{-1 / 2}^{1 / 2} \sigma_{12}\left(w ; \xi_{1}, t\right) d \xi_{1}+\int_{(-1 / 2,-d) \cup(d, 1 / 2)} \sigma_{12}\left(w ; \xi_{1}, 0\right) d \xi_{1} \\
& +\left.\int_{-\pi / 2}^{\pi / 2}\left(\sin \varphi \sigma_{r r}(w ; r, \varphi)+\cos \varphi \sigma_{r \varphi}(w ; r, \varphi)\right) r\right|_{r=d} d \varphi,
\end{aligned}
$$

где $\sigma_{r r}(w)$ и $\sigma_{r \varphi}(w)$ - полярные компоненты тензора напряжений (см., например, $[28$, с. 313$])$.

Для вычисления интегралов $I_{1}^{(t)}$ и $I_{3}^{(d)}$ по верхнему основанию $\Pi_{t, d}$ и по границе полукруга применяем асимптотические формулы (2.25) и (2.28) соответственно. 
Интеграл по нижнему основанию находим, используя краевое условие (2.12). Произведем необходимые выкладки и получим

$$
\begin{aligned}
I_{1}^{(t)}= & \mu a_{1}+O(\exp (-\rho t)), \quad t \rightarrow+\infty, \\
I_{2}^{(0)}= & -\Sigma_{n s}^{0}(s)+o(1), \\
I_{3}^{(d)}= & \frac{1}{2}\left[\lambda\left(1-\frac{1}{\varkappa}\right)(1 \mp 1)+2 \mu\right. \\
& \left.+\mu\left(1-\frac{1}{\varkappa} \pm\left(1+\frac{1}{\varkappa}\right)\right)\right] V_{s}^{(0)}(s) \mathbb{k}(s)+o(1), \quad d \rightarrow+0 .
\end{aligned}
$$

Выполняя предельные переходы $t \rightarrow+\infty$ и $d \rightarrow+0$, выводим из (2.30) и (2.31) искомое равенство (2.26). Наконец, выражение (2.27) для $a_{2}$ получается аналогично, если в формулу Грина подставить $w$ и $\xi_{2} e^{2}$.

ЗАмЕЧАНИЕ 2.2. Перед формулой (2.6) мы постулировали условие $v_{n}=0$ на $\Gamma_{0}$. Теорема 2.1 позволяет подтвердить это предположение. В самом деле, заменив величину $(2.2)$ суммой $w^{0}(s, \xi)+\varepsilon w^{1}(s, \xi)+\cdots$, получим для нового главного члена $w^{0}$ пограничного слоя однородные уравнения $(2.5),(2.3),(2.12)$ и краевое условие

$$
w_{n}^{0}\left(s, \xi_{1}, 0\right)=-v_{n}(s, 0), \quad\left|\xi_{1}\right|<1 / 2
$$

Решением полученной задачи служит вектор $v_{n}(s, 0) e^{2}$, который убывает на бесконечности только при выполнении названного краевого условия на $\Gamma_{0}$.

Нетрудно видеть, что в силу (2.26) и (2.6) предельные краевые условия $(1.13 ;+)$ или $(1.13 ;-)$ обеспечивают равенство нулю коэффициента $a_{1}$ в разложении $(2.25)$. Так как константы $c_{1}$ и $c_{2}$ можно взять произвольными (пусть они нулевые), то теорема 2.1 доставляет ограниченное на бесконечности решение $w$, допускаюшее представление

$$
w(s, \xi)=a_{2}(s) e^{2}+O\left(\exp \left(-\rho \xi_{2}\right)\right)
$$

где $a_{2}-$ величина $(2.27)$.

ЗАМЕЧАНИЕ 2.3. После постановки условий $(1.13 ;+)$ или $(1.13 ;-)$ и выбора констант $c_{1}, c_{2}$ в $(2.24)$ равными нулю мы получили решение $w$, допускаюшее разложение (2.24), в котором величина $C(s)$ фиксирована.

Нам не удалось соблюсти условие экспоненциального затухания пограничного слоя (2.2). Причина состоит в том, что был рассмотрен лишь главный член решения гладкого типа: если вместо $v$ взять сумму $v+\varepsilon v^{1}$, то в правой части $(2.11)$ появится дополнительное слагаемое $v_{n}^{1}(s, 0)$, позволяюшее уничтожить $a_{2}$ за счет выбора значений $v_{n}^{1}(s, 0)$ на $\Gamma_{0}\left(\mathrm{cp}\right.$. с замечанием 2.2). Подчеркнем, что член $a_{2} e^{2}$ не помешает нам в следующем параграфе оценить разность $v-v^{N}$. 


\section{§ 3. Обоснование асимптотики}

1. Глобальное асимптотическое приближение. В $\S 2$ построены асимптотические приближения к решению $v^{N}$ в разных зонах. "Склеим" их в глобальное приближение, пригодное всюду в $\Omega_{N}$.

Пусть $\chi$ - срезаюшая функция, введенная после формулы (2.28). Выберем число $\rho>0$ настолько малым, что функция $\chi_{\rho}$, определенная равенством $\chi_{\rho}(x)=$ $\chi\left(\rho^{-1} n\right)$, обрашается в нуль около гранищы окрестности $\mathscr{U}$, где введены координаты $(s, n)$. Положим еще

$$
\chi^{j}(\varepsilon, x)=\chi\left(\varepsilon^{-2}\left|x-P^{j}\right|\right), \quad X(\varepsilon, x)=1-\sum_{j=1}^{N} \chi^{j}(\varepsilon, x) .
$$

Срезка $\chi_{\rho}$ используется для локализации пограничного слоя, а разбиение единицы (3.1) применяется для срашивания со степенными решениями

$$
K_{j}(\varepsilon) r_{j}^{\mp 1 \pm \pi /\left(2 \alpha_{j}\right)} \Phi^{ \pm}\left(\varphi_{j}\right)
$$

около угловых точек $P^{j}$.

В качестве глобального приближения возьмем вектор-функцию

$$
\begin{aligned}
U^{N}(x)= & X(\varepsilon, x)\left\{v(x)+\varepsilon \chi_{\rho}(x) w\left(s, \varepsilon^{-1} s, \varepsilon^{-1} n\right)\right\} \\
& +\sum_{j=1}^{N} \chi^{j}(\varepsilon, x)\left[v_{s}\left(P^{j}\right)+\varepsilon C\left(P^{j}\right)\right] r^{\mp 1 \pm \pi /\left(2 \alpha_{j}\right)} \Phi^{ \pm}\left(\varphi_{j}\right) .
\end{aligned}
$$

Здесь $v$ и $w$ - решения задач (1.10)-(1.13) и (2.5), (2.3), (2.11), (2.12). В сумме по $j=1, \ldots, N$ коэффициенты $K_{j}(\varepsilon)$ из $(3.2)$ заменяем выражением $V_{s}^{0}\left(P^{j}\right)+\varepsilon C\left(P^{j}\right)$, действуя в соответствии с формулами (2.22)-(2.24) (см. также замечание 2.3$)$. Для того чтобы вектор-функция $U^{N}$ была определена всюду в $\Omega_{N}$, необходимо в случае вогнутого контура продолжить $v$ с $\Omega$ и $w$ с П на более широкие множества. Возможность продолжения решения $v$ на $\Omega \cap \mathscr{U}$ через гладкую границу с сохранением дифференциальных свойств сомнений не вызывает. Благодаря геометрической структуре области $\Omega_{N}$ решение $w$ достаточно распространить на "угловую окрестность" точки 0. Поэтому в (3.3) фигурирует такое продолжение $w$ с полуполосы П на полосу $\left\{\xi:\left|\xi_{1}\right|<1 / 2\right\}$ с разрезом $\left\{\xi: \xi_{1}=0, \xi_{2} \leqslant 0\right\}$, что, во-первых, остается справедливым асимптотическое представление (2.24) и, во-вторых, вне любой окрестности точки $\xi=0$ сохраняется гладкость.

Для обоснования асимптотики мы собираемся подставить $U^{N}$ в краевую задачу (1.5)-(1.7), вычислить возникающие в ней невязки, а затем оценить норму разности $v^{N}-U^{N}$ в “энергетическом" пространстве при помощи вариантов неравенств Корна и Харди. В промежуточных выкладках нам понадобятся весовые классы Кондратьева. Упомянутые неравенства и весовые классы составляют предмет следуюших двух, вспомогательных, пунктов. 
2. Неравенства Корна и Харди. Известно (см. [33]-[35]), что для любой вектор-функции $v$, подчиненной условию (1.2), выполняется соотношение

$$
\mathscr{E}\left(v ; \Omega_{N}\right) \geqslant c\left(\Omega_{N}\right)\left\|v ; W_{2}^{1}\left(\Omega_{N}\right)\right\|^{2},
$$

где левая часть - функционал упругой энергии

$$
\mathscr{E}(v ; \Xi)=\frac{1}{2} \int_{\Xi} \sum_{i, j=1}^{2} \sigma_{i j}(v) \varepsilon_{i j}(v) d x \geqslant \mu \int_{\Xi} \sum_{i, j=1}^{2} \varepsilon_{i j}(v)^{2} d x
$$

а множитель $c\left(\Omega_{N}\right)$ в правой части зависит от области $\Omega_{N}$, но не от $v$. Ближайшая цель - проверить, что величину $c\left(\Omega_{N}\right)$ в $(3.4)$ можно считать не зависящей от параметра $N$.

ПРЕДЛОЖЕНИЕ 3.1. При любом $N$ справедливо неравенство $c\left(\Omega_{N}\right)>c$, где $c$ - положительная постоянная, обусловленная только формой контуров $\Gamma_{0}$ $u \Gamma_{\mathrm{D}}$.

ДокаЗАТЕЛЬСтво. Сначала применим неравенство Корна

$$
\mathscr{E}\left(v ; \Omega \backslash \mathscr{U}_{1}\right) \geqslant c\left(\Omega \backslash \mathscr{U}_{1}\right)\left\|v ; W_{2}^{1}\left(\Omega \backslash \mathscr{U}_{1}\right)\right\|^{2}
$$

в области $\Omega \backslash \mathscr{U}_{1}$, где $\mathscr{U}_{1}$ - (фиксированная) окрестность контура $\Gamma_{0}$, причем $\overline{\mathscr{U}}_{1} \subset \mathscr{U}$ и $\mathscr{U}_{1} \supset \Gamma_{N}$ при $N>N_{0}$ (отметим, что отсутствие вычитаемого const $\left\|v ; L_{2}\left(\Omega \backslash \mathscr{U}_{1}\right)\right\|$ в (3.6) обусловлено соотношениями (1.2)). Множитель $c\left(\Omega \backslash \mathscr{U}_{1}\right)$, разумеется, не зависит от $N$. Осталось исследовать пограничную полоску $\Omega_{N} \cap \mathscr{U}_{1}$.

Пусть $\Gamma_{0}$ - внутренний контур. Разобьем его точками $P_{0}^{1}, \ldots, P_{0}^{2 N_{0}}$ на $2 N_{0}$ дуг равной длины. Выбирая $N_{0}$ большим, добиваемся, чтобы угол $\beta^{i, i+2}$ между внутренними нормалями к $\Gamma_{0}$ в точках $P_{0}^{i}$ и $P_{0}^{i+2}$ удовлетворял условию

$$
0<\beta^{i, i+2}<\frac{\pi}{2}-\delta
$$

где $\delta$ - положительное число.

Из точек $P_{0}^{i}$ и $P_{0}^{i+2}$ проведем лучи вдоль внутренних нормалей, а также касательные к контуру. Так как область, ограниченная $\Gamma_{0}$, является выпуклой и $\beta^{i, i+2}<\pi / 2$, то луч, проведенньй по внутренней нормали в точке $P_{0}^{i}$, и касательная в точке $P_{0}^{i+2}$ пересекаются (то же самое верно по отношению к $P_{0}^{i+2}$ и $P_{0}^{i}$ соответственно).

Фиксируем какой-либо номер $i$ и не будем писать для краткости индексы $i, i+2$ у всех объектов, кроме точек $P_{0}^{i}$ и $P_{0}^{i+2}$. Построим угол с вершиной $P_{0}^{i}$ и раствором $\alpha: \beta<\alpha<\pi / 2$; одна из сторон угла совпадает с лучом, направленным вдоль внутренней нормали, а другая пересекает луч, проведенный из $P_{0}^{i+2}$ по нормали. Выполним аналогичные построения для точки $P_{0}^{i+2}$. Продолжим нормали к $\Gamma_{0}$ в точках $P_{0}^{i}$ и $P_{0}^{i+2}$ за границу области и обозначим точку их пересечения $O$. Выберем из точек $P_{0}^{i}$ и $P_{0}^{i+2}$ ту, для которой расстояние до $O$ максимально. Впишем в угол с этой вершиной и угол с вершиной $O$, образованный нормалями, круг $B_{\alpha}$. Поскольку углы имеют общую сторону, это можно сделать. По построению область $D_{\alpha}$, ограниченная кругом $B_{\alpha}$, дугой $\Gamma_{0}$ и нормалями в точках $P_{0}^{i}$ и $P_{0}^{i+2}$, является 
звездной относительно круга $B_{\alpha}$. Подчеркнем, что любая область $D_{\alpha}^{m}$, ограниченная построенными прямыми, кругом $B_{\alpha}$ и произвольной ломаной $m$, имеющей конщы $P_{0}^{i}$ и $P_{0}^{i+2}$, также является звездной относительно круга $B_{\alpha}$. Ясно, что при уменьшении угла $\alpha$ круг $B_{\alpha}$ удаляется от $\Gamma_{0}$, и можно поместить его вовне окрестности $\mathscr{U}_{1}$.

Вспомогательные построения окончены, и мы, ссылаясь на результаты [28], [29], вправе заключить, что справедлив такой вариант неравенства Корна:

$$
\left\|v ; W_{2}^{1}\left(D_{\alpha}^{m}\right)\right\|^{2} \leqslant c_{1} \mathscr{E}\left(v ; D_{\alpha}^{m}\right)+c_{2}\left\|v ; W_{2}^{1}\left(B_{\alpha}\right)\right\|^{2}
$$

Подчеркнем, что $c_{1}$ и $c_{2}$ не зависят от $m$.

Пусть теперь $\Gamma_{M}$ - произвольная, вписанная в $\Gamma_{0}$ ломаная, причем множество ее вершин $P^{1}, \ldots, P^{M} \supset P^{1}, \ldots, P^{2 N_{0}}$. Ввиду конечности покрытия окрестности $\mathscr{U}_{1} \cap \Omega_{N_{0}}$ множествами $D_{\alpha}$ (перебираются все пары точек $P_{0}^{i}$ и $P_{0}^{i+2}$ ) неравенство (3.8) переносится на множество $\mathscr{U}_{\tau}$, полученное удалением из $\mathscr{U}_{1}$ точек, лежащих внутри $\Gamma_{M}$, т.е. при учете (3.6) оказьвается верньм соотношение

$$
\left\|v ; W_{2}^{1}\left(\mathscr{U}_{\tau}\right)\right\|^{2} \leqslant c\left\{\mathscr{E}\left(v ; \mathscr{U}_{\tau}\right)+\mathscr{E}\left(v ; \Omega \backslash \mathscr{U}_{1}\right)\right\}
$$

Обратимся теперь к области $\Omega_{N}$. Ясно, что при достаточно большом $N$ можно выбирать точки $P_{0}^{1}, \ldots, P_{0}^{2 N_{0}}$ среди вершин ломаной $\Gamma_{N}$. Это обстоятельство заканчивает доказательство для случая многоугольного отверстия. Если же $\Gamma_{0}-$ внешний контур, то все рассуждения только упрощаются.

Следующее утверждение является следствием неравенства (3.4) и вложения $W_{2}^{1}\left(\Omega_{N}\right)$ в $L_{2}\left(\Gamma_{N}\right)$ (независимость постоянной от $N$ вытекает, например, из самого способа доказательства теоремы 1.6.6 из [38]).

ПРЕДЛОЖЕНИЕ 3.2. Для любой $v \in W_{2}^{1}\left(\Omega_{N}\right)$, удовлетворяющей $(1.2)$, справедливо неравенство

$$
\mathscr{E}\left(v ; \Omega_{N}\right) \geqslant c\left\|v ; L_{2}\left(\Gamma_{N}\right)\right\|^{2}
$$

где постоянная с не зависит от $v$ и $N$.

ПРЕДЛОЖЕНИЕ 3.3. Для любой $v \in W_{2}^{1}\left(\Omega_{N}\right)$, удовлетворяющей $(1.2)$, выполняется неравенство

$$
\mathscr{E}\left(v ; \Omega_{N}\right) \geqslant c\left\|\mathscr{R} v ; L_{2}\left(\Omega_{N}\right)\right\|^{2}
$$

где постоянная с не зависит от $v$ u $N=1 / \varepsilon$, весовой множситель $\mathscr{R}$ равен 1 вне обгединения окрестностей $\mathscr{U}$ и $\mathscr{U}_{\mathrm{D}}$ контуров $\Gamma_{N} u \Gamma_{\mathrm{D}}$,

$$
\begin{aligned}
& \mathscr{R}(x)=\operatorname{dist}\left(x, \Gamma_{\mathrm{D}}\right)^{-1}, \quad x \in \mathscr{U}_{\mathrm{D}} \cap \Omega ; \\
& \mathscr{R}(x)=(n+d \varepsilon)^{-1 / 2}(1+|\ln (n+d \varepsilon)|)^{-1}, \quad x \in \mathscr{U} \cap \Omega_{N},
\end{aligned}
$$

причем число $d \geqslant 0$ выбрано так, чтобы $n+d \varepsilon>0$ в $\Omega_{N} \cap \mathscr{U}$ при всех натуральных $N$ (если контур $\Gamma_{0}$ внешний, то можно взять $\left.d=0\right)$. 
ДокАЗАТЕЛЬСТво. Поскольку норма $\left\|v ; L_{2}\left(\Omega_{N}\right)\right\|$ уже оценена (предложение 3.2 ), то умножением на подходящие срезающие функции дело сводится к следуюшим двум ситуациям: $\operatorname{supp} v \subset \mathscr{U}_{\mathrm{D}}$ или $\operatorname{supp} v \subset \mathscr{U}$. В первом случае проверка (3.11) достигается интегрированием вдоль границы известного одномерного неравенства Харди:

$$
4 \int_{0}^{d}\left|\frac{d V}{d n}(n)\right|^{2} d n \geqslant \int_{0}^{d} n^{-2} V(n)^{2} d n \quad \forall V \in C_{0}^{\infty}(0, d]
$$

(разумеется, сначала нужно предположить, что $v_{1}, v_{2} \in C_{0}^{\infty}\left(\mathscr{U}_{\mathrm{D}} \cap \Omega_{N}\right)$, а затем замкнуть полученную оценку по норме пространства $\left.W_{2}^{1}\left(\mathscr{U}_{\mathrm{D}} \cap \Omega_{N}\right)\right)$.

Рассмотрим второй случай (отметим, что в задаче (1.5)-(1.7) не все компоненты решения $v_{N}$ обрашаются в нуль, т.е. применить (3.12) не удается). Установим вспомогательное одномерное неравенство, играющее роль (3.12). Пусть $V \in C_{0}^{\infty}[0, d)$ (теперь функция в точке $n=d$ аннулируется; здесь уместно вспомнить про умножение $v$ на срезку). Имеем

$$
V(t)^{2}=-2 \int_{t}^{d} V(\tau) \frac{d V}{d \tau}(\tau) d \tau
$$

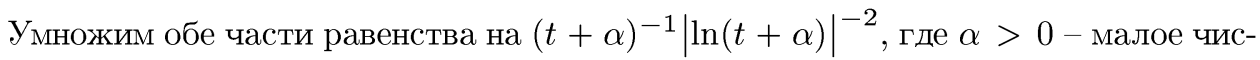
ло, и проинтегрируем его по $t \in(0, d)$. Переставляя пределы интегрирования в повторном интеграле, получаем

$$
\begin{aligned}
& \int_{0}^{d}(t+\alpha)^{-1}|\ln (t+\alpha)|^{-2} V(t)^{2} d t \\
& \quad \leqslant 2 \int_{0}^{d} \int_{t}^{d}(t+\alpha)^{-1}|\ln (t+\alpha)|^{-2}\left|V(\tau) \frac{d V}{d \tau}(\tau)\right| d t d \tau \\
& \quad \leqslant 2 \int_{0}^{d} d \tau\left|V(\tau) \frac{d V}{d \tau}(\tau)\right| \int_{0}^{\tau}(t+\alpha)^{-1}|\ln (t+\alpha)|^{-2} d t \\
& \quad=2 \int_{0}^{d}\left\{[\ln \alpha]^{-1}-[\ln (\tau+\alpha)]^{-1}\right\}\left|V(\tau) \frac{d V}{d \tau}(\tau)\right| d \tau .
\end{aligned}
$$

Выражение в фигурных скобках при $d+\alpha<1$ не превосходит $|\ln (\tau+\alpha)|^{-1} \times$ $(\tau+\alpha)^{-1 / 2}$. Значит,

$$
\int_{0}^{d}(t+\alpha)^{-1}|\ln (t+\alpha)|^{-2} V(t)^{2} d t \leqslant 2 \int_{0}^{d}(\tau+\alpha)^{-1 / 2}|\ln (\tau+\alpha)|^{-1}\left|V(\tau) \frac{d V}{d \tau}(\tau)\right| d \tau
$$

и, разделив на корень из левой части, приходим к нужному неравенству

$$
\int_{0}^{d}(t+\alpha)^{-1}|\ln (t+\alpha)|^{-2} V(t)^{2} d t \leqslant 4 \int_{0}^{d}\left|\frac{d V}{d t}(t)\right|^{2} d t .
$$

Осталось повторить ту же процедуру, что и в случае (3.11); при этом следует учесть, что постоянная в (3.11) не зависит от малого $\alpha>0$, и положить $t=n-\varepsilon d(s)$ и $\alpha=\varepsilon(d+d(s))$, где $n=\varepsilon d(s)-$ уравнение границы $\Gamma_{N}$, a $d>-d(s)$ при всех $s$. 
3. Весовые пространства Кондратьева. В пп. 4 и $2 \S 2$ нам пришлось решать краевую задачу с правой частью, теряюшей гладкость в одной из точек границы, и обсуждать поведение решений около угловых точек. Наиболее приспособленными для этих целей являются весовые классы, введенные В. А. Кондратьевым [25] (см. также [26], [27]); мы уклонились от их использования лишь для упрощения изложения построений (формальных!) в 2 и сейчас восполним этот пробел.

Пусть $\Xi$ - область с кусочно гладкой гранищей; выделенные на $\partial \Xi$ точки $O^{1}, \ldots, O^{J}$, составляющие множество $\mathscr{O}$, разбивают контур $\partial \Xi$ на гладкие дуги, которые встречаются под ненулевыми углами (углы измеряются со стороны $\Xi$ ). Через $V_{\beta}^{l}(\Xi ; \mathscr{O})$ обозначается пространство, полученное пополнением $C_{0}^{\infty}(\bar{\Xi} \backslash \mathscr{O})$ по норме

$$
\left\|w ; V_{\beta}^{l}(\Xi ; \mathscr{O})\right\|=\left(\sum_{k=0}^{l}\left\|\operatorname{dist}(x, \mathscr{O})^{\beta-l+k} \nabla_{x}^{k} w ; L_{2}(\Xi)\right\|^{2}\right)^{1 / 2},
$$

в которой $l=0,1, \ldots ; \beta \in \mathbb{R}$ и $\nabla_{x}^{k} w$ - набор производных порядка $k$ функций $w$. При $l \geqslant 1$ следовое пространство $V_{\beta}^{l-1 / 2}(\partial \Xi ; \mathscr{O})$ снабжается нормой

$$
\left\|v ; V_{\beta}^{l-1 / 2}(\partial \Xi ; \mathscr{O})\right\|=\inf \left\{\left\|w ; V_{\beta}^{l}(\Xi ; \mathscr{O})\right\| \mid v=w \quad \text { на } \partial \Xi \backslash \mathscr{O}\right\} .
$$

Для каждого элемента $v \in V_{\beta}^{l-1 / 2}(\partial \Xi ; \mathscr{O})$, конечно же, имеется продолжение $w \in V_{\beta}^{l}(\Xi ; \mathscr{O})$, подчиненное условию

$$
\left\|w ; V_{\beta}^{l}(\Xi ; \mathscr{O})\right\| \leqslant 2\left\|v ; V_{\beta}^{l-1 / 2}(\partial \Xi ; \mathscr{O})\right\|
$$

При оценке последней нормы удобно применять неравенство

$$
\left\|v ; V_{\beta}^{l-1 / 2}(\partial \Xi ; \mathscr{O})\right\| \leqslant c\left\|v ; V_{\beta+1 / 2}^{l}(\partial \Xi ; \mathscr{O})\right\|,
$$

причем норма в $V_{\beta}^{l}(\partial \Xi ; \mathscr{O})$ получается простой заменой в $(3.14)$ области $\Xi$ ее границею $\partial \Xi$. (По поводу всех определений и сформулированных фактов см. [25] или [27].)

Приведем дополнительно теорему 3.4 о разрешимости задачи $(2.5),(2.3),(2.11)$, (2.12) в весовых классах. Первая ее часть по существу уже использовалась при проверке утверждения 2.1 - в ней применяются пространства с экспоненциальными весовыми множителями, обслуживающими бесконечно удаленную точку (уместно заметить, что заменой $\xi_{2} \mapsto \rho=\exp \left(-\xi_{2}\right)$ полуполоса $\Pi=\left\{\xi: \xi_{2}>0\right.$, $\left.\left|\xi_{1}\right|<1 / 2\right\}$ переводится в сектор, а соответствующий весовой класс становится кондратьевским). Во второй части точка $\xi=0$ рассматривается как угловая, и потому в нормах функциональных пространств возникают те же весовые множители, что и в (3.14). Доказательство теоремы получается прямыми ссылками на общие результаты $[27$, утверждения 5.1.4, 5.4.12, 6.1.7, 5.4.4].

Укажем список нужных функциональных пространств: $\mathscr{W}_{\gamma}^{l}(\Pi ; \infty)$ - пополнение линеала $\mathscr{C}_{0}^{\infty}(\bar{\Pi})$ функций из $C_{0}^{\infty}(\bar{\Pi})$, подчиненных условиям периодичности на боковых сторонах $\pi_{ \pm}$полуполосы П, по норме

$$
\left\|w ; \mathscr{W}_{\gamma}^{l}(\Pi ; \infty)\right\|=\left(\sum_{k=0}^{l}\left\|\exp \left(\gamma \xi_{2}\right) \nabla_{\xi}^{k} w ; L_{2}(\Pi)\right\|^{2}\right)^{1 / 2}
$$


$\mathscr{V}_{\gamma, \beta}^{l}(\Pi ; \infty, 0)-$ пополнение $\mathscr{C}_{0}^{\infty}(\bar{\Pi})$ по норме

$$
\left\|w ; \mathscr{V}_{\gamma, \beta}^{l}(\Pi ; \infty, 0)\right\|=\left(\sum_{k=0}^{l}\left\|\min \{1,|\xi|\}^{\beta-l+k} \exp \left(\gamma \xi_{2}\right) \nabla_{\xi}^{k} w ; L_{2}(\Pi)\right\|^{2}\right)^{1 / 2} ;
$$

$\mathscr{V}_{\gamma, \beta}^{l+1,1}(\Pi ; \infty, 0)$ имеет следующую норму:

$$
\left(\left\|w ; \mathscr{V}_{\gamma, \beta-l}^{0}(\Pi ; \infty, 0)\right\|^{2}+\left\|\nabla_{\xi} w ; \mathscr{V}_{\gamma, \beta}^{l}(\Pi ; \infty, 0)\right\|^{2}\right)^{1 / 2}
$$

соответствующие следовые пространства наделяются естественной нормой (ср. с (3.15)); при этом присутствие весового множителя на бесконечности, управляемого индексом $\gamma$, никак не сказывается на определении следа функции на торце полуполосы.

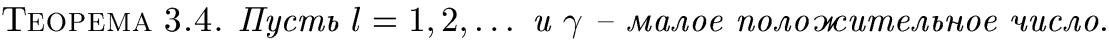

1) Oператорь $A_{+}$и $A_{-}$задачи (2.5), (2.3), (2.11), (2.12),

$$
\begin{aligned}
\mathscr{W}_{ \pm \gamma}^{l+1}(\Pi ; \infty)^{2} & \ni w \mapsto A_{ \pm} w=\left\{-L w,\left.w_{2}\right|_{\pi_{0}},\left.\mu\left(\frac{\partial w_{1}}{\partial \xi_{2}}+\frac{\partial w_{2}}{\partial \xi_{1}}\right)\right|_{\pi_{0}}\right\} \\
& \in \mathscr{W}_{ \pm \gamma}^{l-1}(\Pi ; \infty)^{2} \times \mathscr{W}_{ \pm \gamma}^{l+1 / 2}\left(\pi_{0} ; \infty\right) \times \mathscr{W}_{ \pm \gamma}^{l-1 / 2}\left(\pi_{0} ; \infty\right)
\end{aligned}
$$

являются соответственно мономорфизмом и әпиморфизмом, причем

$$
\begin{aligned}
\operatorname{ker} A_{-} & =\left\{c_{1} e^{1}+c_{2} \xi_{2} e^{2}: c_{i} \in \mathbb{R}\right\}, \\
\operatorname{coker} A_{+} & =\left\{\left(v,-\sigma_{22}(v), v_{1}\right): v \in \operatorname{ker} A_{-}\right\} .
\end{aligned}
$$

2) Если $\beta \in(l-1, l)$, то все сказанное в $n .1)$ об операторах (3.18) остается в силе и для операторов

$$
\begin{gathered}
\mathscr{V}_{ \pm \gamma, \beta}^{l+1,1}(\Pi ; \infty, 0) \times \mathcal{V}_{ \pm \gamma, \beta}^{l+1}(\Pi ; \infty, 0) \ni w=\left(w_{1}, w_{2}\right) \\
\mapsto A_{ \pm} w=\left\{-L w,\left.w_{2}\right|_{\pi_{0}},\left.\mu\left(\frac{\partial w_{1}}{\partial \xi_{2}}+\frac{\partial w_{2}}{\partial \xi_{1}}\right)\right|_{\pi_{0}}\right\} \\
\in \mathscr{V}_{ \pm \gamma, \beta}^{l-1}(\Pi ; \infty, 0)^{2} \times \mathscr{V}_{ \pm \gamma, \beta}^{l+1 / 2}\left(\pi_{0} ; \infty, 0\right) \times \mathscr{V}_{ \pm \gamma, \beta}^{l-1 / 2}\left(\pi_{0} ; \infty, 0\right) .
\end{gathered}
$$

Сведений, перечисленных в теореме 3.4 , достаточно для того, чтобы сделать все необходимые заключения о свойствах пограничного слоя, однако, по мнению авторов, в $\S 2$ был избран более конструктивный и наглядный путь.

4. Невязка в первом из условий (1.7). Найдем правые части уравнений $(1.5)-(1.7)$, которым удовлетворяет разность $v^{N}-U^{N}$. Начнем с краевых условий. По построению (см. (1.9) и $(3.3),(3.1)) U^{N}=0$ на $\Gamma_{\mathrm{D}}$, и потому в силу $(1.2)$

$$
v^{N}(x)-U^{N}(x)=0, \quad x \in \Gamma_{\mathrm{D}} .
$$

Обратимся теперь к краевым условиям на ломаной $\Gamma_{N}$. Пусть $\gamma^{01}-$ ее звено, соединяюшее точки $P^{0}$ и $P^{1}$. Достаточно рассматривать часть $\gamma_{0}$ звена, примыкающую к $P^{0}$ (с другой частью $\gamma_{1}$, равно как и с остальными звеньями, поступаем аналогично). 
Согласно определениям угловых частей в $(2.17) \Phi_{\varphi}^{ \pm}\left(\alpha_{j}\right)=0$ и, значит, на $\gamma_{0}$ выполняется равенство

$$
U^{N}(x) \cdot \nu(x)=X(\varepsilon, x)\left\{v(x) \cdot \nu(x)+\varepsilon w\left(s, \varepsilon^{-1} s, \varepsilon^{-1} n\right) \cdot \nu(x)\right\}
$$

где $\nu$ - нормаль к $\gamma$. Перейдем в (3.20) к быстрым переменным (2.1) - для обозначения этого перехода будем писать знак “ " над символами $\gamma, \nu, v$ и т.п. В силу (2.6) и (2.8) заключаем, что символ $O(\varepsilon)$, оканчивающий формулу $(2.9)$, можно истолковать как соотношение

$$
\left\|\tilde{v} \cdot \tilde{\nu}+\varepsilon f ; V_{1+\delta}^{3 / 2}\left(\tilde{\gamma}_{0} ; \widetilde{P}^{0}\right)\right\| \leqslant c_{\delta} \varepsilon^{2}
$$

где $\delta$ - произвольное положительное число, а $f\left(\xi_{1}\right)$ - правая часть (2.11). Кроме того, учитывая $(2.7),(2.8)$ и (2.11), а также сказанное в конще п. 1 о продолжении решения $w$, приходим к оценке

$$
\left\|\widetilde{w} \cdot \tilde{\nu}-f ; V_{1+\delta}^{3 / 2}\left(\tilde{\gamma}_{0} ; \widetilde{P}^{0}\right)\right\| \leqslant c_{\delta} \varepsilon|\ln \varepsilon| .
$$

Подчеркнем, что при выводе (3.20) и (3.21) мы использовали неравенство (3.17) при $l=2, \beta=1+\delta$ и имели дело с пространствами, снабженными цельми индексами $l$.

Итак, в соответствии со сказанным в п.3 существует продолжение $\tilde{y}_{\nu}^{0}$ в классе $V_{1+\delta}^{2}$ выражения, выделенного фигурными скобками в (3.20). Это продолжение необходимо умножить на $\widetilde{X}$ (ср. с левой частью (3.20)). С целью согласовать построения, проводимые около вершины $P^{0}$ на части звена $\gamma_{0}$, с рассмотрением других звеньев и вершины ломаной $\Gamma_{N}$ добавим еше два множителя: $\chi\left(\pi^{-1} \varphi_{0}+1 / 2\right)$ и $1-\chi(|\xi| / 2)$; здесь $\varphi_{0}-$ угловая переменная, введенная в начале п. $3 \S 2$, а $\chi$ - срезающая функция, описанная после формулы (2.28). В результате оказывается, что носитель нового продолжения (обозначим его $\widetilde{Y}_{\nu}^{0}$ ) содержится в секторе

$$
\widetilde{K}^{0}=\left\{\xi:|\xi|<2 / 3, \quad \varphi_{0} \in[-\pi / 2,-\pi / 6]\right\} .
$$

Так как $\chi\left(\pi^{-1} \varphi_{0}+1 / 2\right)=1$ при $\varphi_{0} \in[-\pi / 2,-\pi / 3]$, то след $\tilde{Y}_{\nu}^{0}$ равен $\widetilde{X} \tilde{y}_{\nu}^{0}(1-\chi)$, причем благодаря множителю $1-\chi(|\xi| / 2)$ продолжение $\tilde{Y}_{\nu}^{0}$ можно гладко "склеить" с продолжением $\widetilde{Y}_{\nu}^{1}$, обслуживающим другую часть $\gamma_{1}$ того же звена (и вершину $\left.P^{1}\right)$. Кроме того,

$$
\begin{aligned}
& \left\|\widetilde{Y}_{\nu}^{0} ; V_{1+\delta}^{2}\left(\widetilde{K}^{0} ; \widetilde{P}^{0}\right)\right\| \leqslant c\left\|\tilde{y}_{\nu}^{0} ; V_{1+\delta}^{2}\left(\widetilde{K}^{0} ; \widetilde{P}^{0}\right)\right\| \\
& \quad \leqslant c\left\{\left\|\tilde{v} \cdot \tilde{\nu}+\varepsilon f ; V_{1+\delta}^{3 / 2}\left(\tilde{\gamma}_{0} ; \widetilde{P}^{0}\right)\right\|+\varepsilon\left\|\tilde{w} \cdot \tilde{\nu}-f ; V_{1+\delta}^{3 / 2}\left(\tilde{\gamma}_{0} ; \widetilde{P}^{0}\right)\right\|\right\} \leqslant c_{\delta} \varepsilon^{2}|\ln \varepsilon| .
\end{aligned}
$$

В пояснении нуждается только первое из указанных неравенств - остальные суть следствия соотношений $(3.15)$ и $(3.21),(3.22)$. Функщия $\widetilde{Y}_{\nu}^{0}$ отличается от $\tilde{y}_{\nu}^{0}$ лишь срезками. Так как $1-\chi(|\xi| / 2)=0$ вблизи вершины $\widetilde{P}^{0}$, то умножение на такую срезку прибавляет к норме выражение, мажорируемое величиной

$$
c\left\|\tilde{y}_{\nu}^{0} ; W_{2}^{1}\left(\left\{\xi \in \widetilde{K}^{0}:|\xi|>1 / 3\right\}\right)\right\|
$$


которая, конечно же, допускает нужную оценку. Учитывая очевидные неравенства

$$
\left|\nabla_{\xi}^{k} \chi\left(\pi^{-1} \varphi_{0}+1 / 2\right)\right| \leqslant c_{k}|\xi|^{-k}
$$

и распределение степеней весовых множителей в (3.14), заключаем, что срезающая функция, зависящая только от угловой переменной, является мультипликатором в $V_{1+\delta}^{2}$, т.е. неравенство (3.23) нарушиться не может. Наконец, согласно определениям (3.1)

$$
\operatorname{supp}\left(\nabla_{\xi}\left(1-\tilde{\chi}^{0}\right)\right) \subset\{\xi: \varepsilon \leqslant|\xi| \leqslant \varepsilon / 3\}, \quad\left|\nabla_{\xi}^{k}\left(1-\tilde{\chi}^{0}\right)\right| \leqslant c_{k} \varepsilon^{-k}
$$

и, следовательно, верна формула $\left|\nabla_{\xi}^{k}\left(1-\tilde{\chi}^{0}\right)\right| \leqslant c_{k}|\xi|^{-k}$, сохраняюшая предыдущую аргументацию при рассмотрении множителя $\widetilde{X}$.

Продолжим преобразования. Поскольку $\widetilde{Y}_{\nu}^{0}=0$ при $|\xi|<\varepsilon / 6$, то, отшепив от весового множителя в норме пространства $V_{1+\delta}^{2}$ выражение $\operatorname{dist}\left(\xi, \widetilde{P}^{0}\right)^{\delta}=|\xi|^{\delta}$, большее $c \varepsilon^{\delta}$ на $\operatorname{supp} \widetilde{Y}_{\nu}^{0}$, получаем

$$
\left\|\widetilde{Y}_{\nu}^{0} ; V_{1+\delta}^{2}\left(\widetilde{K}^{0} ; \widetilde{P}^{0}\right)\right\| \geqslant c \varepsilon^{\delta}\left\|\widetilde{Y}_{\nu}^{0} ; V_{1}^{2}\left(\widetilde{K}^{0} ; \widetilde{P}^{0}\right)\right\|
$$

(отметим, что $V_{1}^{2}\left(\widetilde{K}^{0} ; \widetilde{P}^{0}\right) \subset W_{2}^{1}\left(\widetilde{K}^{0}\right)$ в соответствии с $\left.(3.14)\right)$. Таким образом, загрубив (3.23), приходим к оценке

$$
\left\|\widetilde{Y}_{\nu}^{0} ; V_{1}^{2}\left(\widetilde{K}^{0} ; \widetilde{P}^{0}\right)\right\| \leqslant c \varepsilon^{2(1-\delta)} .
$$

Вернемся к исходным, медленным, переменным (в принятых обозначениях это равносильно снятию символа “ ”). Несложные вычисления, используюшие (3.14), показывают, что норма в $V_{1}^{2}$ инвариантна относительно растяжения координат (удачное согласование индексов $\beta$ и $l$ с размерностью области). Значит,

$$
\left\|Y_{\nu}^{0} ; V_{1}^{2}\left(K^{0} ; P^{0}\right)\right\| \leqslant c \varepsilon^{2(1-\delta)} .
$$

Подобное неравенство выполняется и для $Y_{\nu}^{1}$. След суммы $Y_{\nu}^{01}=Y_{\nu}^{0}+Y_{\nu}^{1}$ на звене $\gamma^{01}$, соединяющем $P^{0}$ и $P^{1}$, совпадает со следом величины $(3.20)$, а носитель расположен в тупоугольном равнобедренном треугольнике с основанием $\gamma^{01}$, причем $Y_{\nu}^{01}=0$ на боковых сторонах и в $c \varepsilon^{2}$-окрестностях вершин $P^{0}$ и $P^{1}$ (см. пояснения, предшествовавшие (3.23)).

Определим теперь векторное поле $Y^{01}=\left(Y_{1}^{01}, Y_{2}^{01}\right)$ так, чтобы

$$
Y^{01} \cdot \nu=Y_{1}^{01} \nu_{1}+Y_{2}^{01} \nu_{2}=Y_{\nu}^{01}, \quad Y_{1}^{01} \nu_{2}-Y_{2}^{01} \nu_{1}=0
$$

и продолжим его нулем с треугольника на всю область $\Omega_{N}$. Из $(3.24)$ и аналогичного неравенства для $Y_{\nu}^{1}$ выводим

$$
\left\|Y^{01} ; V_{1}^{2}\left(\Omega_{N} ; \mathscr{P}\right)\right\|^{2} \leqslant c_{\delta} \varepsilon^{4(1-\delta)} .
$$

Повторим проведенные построения для звеньев $\gamma^{12}, \ldots, \gamma^{N-1,0}$. Найдены продолжения $Y^{01}, Y^{12}, \ldots, Y^{N-1,0}$, сумма которых компенсирует невязку функции $U^{N}$ в первом из краевых условий (1.7) (обращаем внимание на то, что их носители попарно дизъюнктны). Для каждой из вектор-функций $Y^{j, j+1}$ справедливо неравенство типа (3.25), их количество равно $N$, и потому

$$
\left\|Y ; V_{1}^{2}\left(\Omega_{N} ; \mathscr{P}\right)\right\| \leqslant c_{\delta} \varepsilon^{(3-4 \delta) / 2} .
$$

Отметим еще, что, как уже упоминалось, $Y \in W_{2}^{1}\left(\Omega_{N}\right)$ и

$$
\left\|Y ; W_{2}^{1}\left(\Omega_{N}\right)\right\| \leqslant c\left\|Y ; V_{1}^{2}\left(\Omega_{N} ; \mathscr{P}\right)\right\| \leqslant c_{\delta} \varepsilon^{(3-4 \delta) / 2} .
$$


5. Невязка во втором из условий (1.7). Как и п. 4, нас будет интересовать часть $\gamma_{0}$ звена $\gamma^{01}$. Согласно $(2.6),(2.10)$ и $(2.8)$

$$
\left\|\sigma_{\nu \tau}(v)-\Sigma_{n s}^{0} ; L_{2}\left(\gamma_{0}\right)\right\| \leqslant c \varepsilon^{3 / 2}
$$

причем лишний множитель $\varepsilon^{1 / 2}$ появился в связи с малостью длины $\gamma_{0}$. В (3.3) решение $v$ умножается на срезающую функцию $X$, и при вычислении $\sigma_{\nu \tau}(X v)$ ее нужно дифференцировать. Так как координаты $(\nu, \tau)$ декартовы (дуга $\gamma_{0}$ прямолинейна) и $X$ вблизи $P^{0}$ зависит только от $r_{0}$, то на $\gamma_{0}$

$$
\sigma_{\nu \tau}(X v)=X \mu\left(\frac{\partial v_{\nu}}{\partial \tau}+\frac{\partial v_{\tau}}{\partial \nu}\right)+\mu v_{\nu} \frac{\partial X}{\partial \tau}=X \sigma_{\nu \tau}(v)-\mu v_{\nu} \frac{\partial \chi^{0}}{\partial r_{0}}
$$

(ср. с формулами для $\sigma_{i j}$ в п. $1 \S 1$ и см. (3.1)). На носителе $\partial \chi^{0} / \partial r_{0}$ имеем $n=$ $O\left(\varepsilon^{2}\right)$ и $|\xi|=O(\varepsilon)$, т.е. в силу $(2.8)$

$$
\nu_{n}(x)=1+O(\varepsilon), \quad \nu_{s}(x)=-\frac{\varepsilon}{2} \mathbb{k}\left(P^{0}\right)+O\left(\varepsilon^{2}\right) .
$$

Таким образом, вспоминая (2.6), получаем соотношения

$$
v_{\nu}(x)=v_{n}(x) \nu_{n}(x)+v_{s}(x) \nu_{s}(x)=-\frac{\varepsilon}{2} \mathbb{k}\left(P^{0}\right) v_{s}\left(P^{0}\right)+O\left(\varepsilon^{2}\right) .
$$

Поэтому в соответствии с (3.1)

$$
\left\|\frac{\partial \chi^{0}}{\partial r_{0}}\left[v_{\nu}+\frac{\varepsilon}{2} \mathbb{k}\left(P^{0}\right) v_{s}\left(P^{0}\right)\right] ; L_{2}\left(\gamma_{0}\right)\right\|^{2} \leqslant c \int_{c \varepsilon^{2}}^{C \varepsilon^{2}}\left|\varepsilon^{2} \frac{1}{\varepsilon^{2}}\right|^{2} d \tau=c \varepsilon^{2}
$$

(при использовании весовых классов можно получить асимптотически точную оценку, но нас устроит и (3.29); ср. далее с замечанием 3.5). Окончательно

$$
\left\|\sigma_{\nu \tau}(X v)-X \Sigma_{n s}^{0}-\mu \frac{\varepsilon}{2} \mathbb{k}\left(P^{0}\right) v_{s}\left(P^{0}\right) \frac{\partial \chi^{0}}{\partial r_{0}} ; L_{2}\left(\gamma_{0}\right)\right\|^{2} \leqslant c \varepsilon^{2} .
$$

Аналогичные преобразования нужно проделать и с пограничным слоем (2.2). Согласно (2.23) (или (2.28)) вблизи $P^{0}$ запишем $w$ в виде

$$
w(s, \xi)=W^{0}\left(r_{0}, \varphi_{0}\right)+W(s, \xi),
$$

где

$$
\begin{aligned}
W(s, \xi) & =O(|\xi|[1+|\ln | \xi||]), \quad\left|\nabla_{\xi} W(s, \xi)\right|=O(1+|\ln | \xi||), \\
W^{0}(r, \varphi) & =\pi^{-1} \mathbb{k}\left(P^{0}\right) v_{s}\left(P^{0}\right)\left[ \pm \Phi^{1}(\varphi, \ln r)-\Psi(\varphi)\right]+\varepsilon C\left(P^{0}\right) \Phi^{0}(\varphi) .
\end{aligned}
$$

Используя $(2.7),(2.8),(2.12)$ и формулу вида (3.28), заключаем, что

$$
\left\|\varepsilon \sigma_{\nu \tau}(X v)+X \Sigma_{n s}^{0}+\mu \varepsilon w_{\nu} \frac{\partial \chi^{0}}{\partial r_{0}} ; L_{2}\left(\gamma_{0}\right)\right\| \leqslant c \varepsilon^{3 / 2}|\ln \varepsilon| .
$$


Приведенные оценки для остатка в представлении (3.31) показывают, что

$$
\mu \varepsilon\left\|\left(w_{\nu}-W_{\nu}^{0}\right) \frac{\partial \chi^{0}}{\partial r_{0}} ; L_{2}\left(\gamma_{0}\right)\right\|^{2} \leqslant c \varepsilon^{2}|\ln \varepsilon| .
$$

Кроме того, принимая во внимание явные выражения для угловых частей $\Phi^{i}$ и $\Psi$ (см. п. $2 \oint 2$ ), находим

$$
\begin{aligned}
-W_{\nu}^{0}\left(r_{0},-\alpha_{0}\right) & =W_{\varphi}^{0}\left(r_{0},-\alpha_{0}\right) \\
& =\pi^{-1} \mathbb{k}\left(P^{0}\right) v_{s}\left(P^{0}\right)\left[ \pm \Phi_{\varphi}^{1}\left(-\alpha_{0}, \ln r\right)-\Psi_{\varphi}\left(-\alpha_{0}\right)\right]+\varepsilon C\left(P^{0}\right) \Phi_{\varphi}^{0}\left(-\alpha_{0}\right) \\
& =-\pi^{-1} \mathbb{k}\left(P^{0}\right) v_{s}\left(P^{0}\right) \alpha_{0} \sin \alpha_{0}+O(\varepsilon),
\end{aligned}
$$

т.е. после учета (2.15)

$$
W_{\nu}^{0}\left(r_{0}, \alpha_{0}\right)=v_{s}\left(P^{0}\right) \mathbb{k}\left(P^{0}\right) / 2+O(\varepsilon) .
$$

Следовательно,

$$
\left\|\varepsilon \sigma_{\nu \tau}(X w)+X \Sigma_{n s}^{0}+\mu \frac{\varepsilon}{2} \mathbb{k}\left(P^{0}\right) v_{s}\left(P^{0}\right) \frac{\partial \chi^{0}}{\partial r_{0}} ; L_{2}\left(\gamma_{0}\right)\right\| \leqslant c \varepsilon^{2}|\ln \varepsilon|^{2} .
$$

Наконец, в силу формул для специальных решений (2.17) напряжения

$$
\sigma_{\nu \tau}\left(\chi^{0}\left(\varepsilon^{-2} r_{0}\right) r_{0}^{\mp 1 \pm \pi /\left(2 \alpha_{0}\right)} \Phi^{ \pm}\left(\varphi_{0}\right)\right)
$$

аннулируются при $\varphi_{0}=-\alpha_{0}$. Подводя итог, напишем, что согласно $(3.30)$ и

$$
\left\|\sigma_{\nu \tau}\left(U^{N}\right) ; L_{2}\left(\gamma_{0}\right)\right\|^{2} \leqslant c \varepsilon^{2}|\ln \varepsilon|^{2}
$$

Получив аналогичные неравенства для $\gamma_{1}$ и для остальных звеньев ломаной, придем к требуемой оценке

$$
\left\|\sigma_{\nu \tau}\left(U^{N}\right) ; L_{2}\left(\Gamma_{N}\right)\right\|^{2} \leqslant c N \varepsilon^{2}|\ln \varepsilon|^{2}=c \varepsilon|\ln \varepsilon|^{2} .
$$

6. Невязка в уравнении (1.5). Используя равенство (1.8) и тот факт, что векторы (2.17) суть решения однородной системы Ламе, получаем соотношение

$$
\begin{aligned}
L U^{N}-f= & -\sum_{j=1}^{N} \chi^{j} f-\sum_{j=1}^{N}\left[L, \chi^{j}\right]\left\{v-\left(v_{s}\left(P^{j}\right)\right.\right. \\
& \left.\left.+\varepsilon C\left(P^{j}\right)\right) r_{j}^{\mp 1 \pm \pi /\left(2 \alpha_{j}\right)} \Phi^{ \pm}\left(\varphi_{j}\right)\right\}+\varepsilon L X \chi_{\rho} w
\end{aligned}
$$

в котором $[A, B]=A B-B A-$ коммутатор операторов $A$ и $B$.

Рассмотрим последнее слагаемое в (3.35). Согласно (2.32) запишем решение типа пограничного слоя в виде

$$
w(s, \xi)=\mathbf{n} a_{2}(s)+w^{0}(s, \xi)
$$

где $a_{2}$ - гладкая функция переменной $s$, а решение $w^{0}(s, \xi)$ системы $(2.5)$ экспоненциально затухает при $\xi_{2} \rightarrow+\infty$. (В связи с новой записью слагаемого в $(3.36)$ 
напомним, что в $\oint 2$ были приняты специальные обозначения для $w_{s}$ и $w_{n}$; см. комментарии к (2.5).) Вынесем срезающую функцию $\chi_{\rho}$ за оператор $L$ и заметим, что согласно ее определению носители коэффищиентов коммутатора $\left[L, \chi_{\rho}\right]$ удалены на расстояние $\rho / 6$ от контура $\Gamma_{0}$. Поэтому невязка, возникающая из-за составляющей $w^{0}$, экспоненциально мала, и верна оценка

$$
\varepsilon\left\|\left[L, \chi_{\rho}\right] X w ; L_{2}\left(\Omega_{N}\right)\right\|=\varepsilon\left\|\left[L, \chi_{\rho}\right] w ; L_{2}\left(\Omega_{N}\right)\right\| \leqslant c \varepsilon .
$$

Далее имеем

$$
\varepsilon \chi_{\rho} L X w=\varepsilon \chi_{\rho} X L w-\varepsilon \sum_{j=1}^{N}\left[L, \chi^{j}\right] w .
$$

Опять-таки

$$
\varepsilon\left\|\chi_{\rho} X L \mathbf{n} a_{2} ; L_{2}\left(\Omega_{N}\right)\right\| \leqslant c \varepsilon,
$$

т.е. нужно обработать величину $\varepsilon \chi_{\rho} X L w^{0}$. В п. $1 \S 2$ при выводе уравнений $(2.5)$ из системы Ламе (2.4), записанной в криволинейных координатах, были учтены все выражения, содержащие вторые производные $\partial^{2} / \partial \xi_{i} \partial \xi_{j}$ с коэффициентами, ограниченными при $n \rightarrow 0$. Значит, разделяя быстрые и медленные переменные, выводим представление

$$
L\left(\nabla_{x}\right) w(s, \xi)=\mathfrak{L}\left(s, n, \nabla_{\xi}, \partial_{s}\right) w^{0}(s, \xi)
$$

где $\mathfrak{L}$ - оператор, содержащий следующие дифференциальные выражения с ограниченными коэффициентами:

$$
n \partial^{2} / \partial \xi_{i} \partial \xi_{j}, \quad \partial / \partial \xi_{i}, \quad 1, \quad \partial^{2} / \partial s^{2}, \quad \partial / \partial s .
$$

Следовательно,

$$
\begin{aligned}
& \varepsilon\left\|n^{1 / 2}(1+|\ln | n||) \chi_{\rho} X L w^{0} ; L_{2}\left(\Omega_{N}\right)\right\| \\
& \quad \leqslant c \varepsilon\left[\sum_{i=0}^{2} \varepsilon^{-2 i} \int_{c \varepsilon}^{\infty} n^{1+2 \delta_{i, 2}}(1+|\ln | n||)^{2} e^{-\delta n / \varepsilon} d n\right]^{1 / 2} \leqslant c \varepsilon|\ln \varepsilon| .
\end{aligned}
$$

Подчеркнем, что здесь весовой множитель взят в соответствии с неравенством Харди (3.11). Поскольку $n=O\left(\varepsilon^{2}\right)$ на supp $\chi^{j}$ (см. (3.1)), то этот же вес позволяет получить приемлемую оценку для первой суммы справа в (3.35):

$$
\left\|n^{1 / 2}(1+|\ln | n||) \sum_{j=1}^{N} \chi^{j} f ; L_{2}\left(\Omega_{N}\right)\right\| \leqslant c \varepsilon|\ln \varepsilon| \times\left\|f ; L_{2}\left(\Omega_{N}\right)\right\| .
$$

Итак, осталось рассмотреть выражения

$$
\begin{gathered}
\Upsilon^{j}=\left[L, \chi^{j}\right]\left\{v+\varepsilon w-\left[v_{s}\left(P^{j}\right)+C\left(P^{j}\right)\right] r^{\mp 1 \pm \pi /\left(2 \alpha_{j}\right)} \Phi^{ \pm}\left(\varphi_{j}\right)\right\} \\
j=1, \ldots, N
\end{gathered}
$$

носители которых расположены в кольцах $\left\{x: \varepsilon^{2} / 6 \leqslant r_{j} \leqslant \varepsilon^{2} / 3\right\}$. Сначала заменим в (3.37) $v$ на $\mathbf{s} v_{s}\left(P^{j}\right)$ и $\varepsilon w$ на $\varepsilon W^{0}\left(P^{j}, r_{j}, \varphi_{j}\right)$ (см. (3.32)); при этом ввиду малости остатков в асимптотических представлениях (2.6) и (3.31) мы совершим ошибку 
$O(\varepsilon|\ln \varepsilon|)$. Затем применим разложения (2.19) и аналогичные разложения градиентов степенных решений (2.17) (последние можно получить формальным дифференцированием (2.19)); при этом согласно (2.14) и (2.15) величина $\delta$ в (2.19) равна $\varepsilon\left|\mathbb{k}\left(P^{j}\right)\right|$. В результате оказывается, что основные члены асимптотики взаимно уничтожаются, а преобразованное выражение (3.37) после выполнения дифференцирований, предписанных коммутатором $\left[L\left(\nabla_{x}\right), \chi^{j}\right]$, становится равным

$$
O\left(\varepsilon^{2}|\ln r|^{2}\left\{\varepsilon^{-2} r^{-1}+\varepsilon^{-4}\right\}\right)
$$

(отметим, что каждое дифференцирование срезки $\chi^{j}$ приводит к появлению множителя $\varepsilon^{-2}$; см. (3.1)). Окончательно

$$
\begin{aligned}
& \left\|n^{1 / 2}(1+|\ln | n||) \sum_{j=1}^{N} \Upsilon^{j} ; L_{2}\left(\Omega_{N}\right)\right\|^{2}=\sum_{j=1}^{N}\left\|n^{1 / 2}(1+|\ln | n||) \Upsilon^{j} ; L_{2}\left(\Omega_{N}\right)\right\|^{2} \\
& \quad \leqslant c \sum_{j=1}^{N} \varepsilon^{-4} \int_{c \varepsilon^{2}}^{C \varepsilon^{2}} r(1+|\ln r|)^{2}|\ln r|^{4}\left[1+\varepsilon^{2} r^{-2}\right]^{2} r d r \\
& \quad \leqslant c \sum_{j=1}^{N} \varepsilon^{2}|\ln \varepsilon|^{6} \leqslant c \varepsilon|\ln \varepsilon|^{6} .
\end{aligned}
$$

Собирая полученные оценки, приходим к искомому неравенству

$$
\left\|\mathscr{R}^{-1}\left(L U^{N}-f\right) ; L_{2}\left(\Omega_{N}\right)\right\| \leqslant c \varepsilon^{1 / 2}|\ln \varepsilon|^{3} ;
$$

здесь $\mathscr{R}$ - весовой множитель, введенный в предложении 3.2 .

ЗАмЕчАниЕ 3.5. Порядок мажоранты в (3.39) определяется самой грубой из оценок слагаемых в (3.35). В данном пункте таковой оказалась оценка (3.38). Этого и следовало ожидать, поскольку применялся стандартньй, но неточньй прием срашивания разложений в "промежуточной" зоне - при помоши разбиения единицы (3.1). Его можно усовершенствовать (см. $[39, \S 2.1])$ и заменить правую часть (3.38) величиной $c \varepsilon^{2}|\ln \varepsilon|^{6}$, однако для основных целей статьи такое улучшение необязательно, и мы отдаем предпочтение простоте асимптотической конструкции.

7. Оправдание асимптотики. В настоящем пункте будет установлено следуюшее утверждение, обосновываюшее асимптотические построения.

Теорема 3.6. При выполнении условий, перечисленных в $n .1 \S 1$, для решения $v^{N}$ задачи (1.5)-(1.7) справедливо неравенство

$$
\left\|v^{N}-U^{N} ; W_{2}^{1}\left(\Omega_{N}\right)\right\| \leqslant c_{\delta} \varepsilon^{(1-2 \delta) / 2},
$$

в котором $U^{N}$ - глобальное асимптотическое приближение, определенное формулой (3.3) по решениям $v$ и ш задач (1.8)-(1.11) и (2.5), (2.3), (2.11), (2.12) (см. $\S 2), \delta$ - произвольное положительное число, а величина $c_{\delta}$ зависит от $\delta$, но не от $\varepsilon=1 / N$. 
ДоКАЗАТЕЛЬСТво. Ввиду оценки (3.27) проверку соотношения (3.40) можно заменить проверкой соотношения

$$
\left\|R^{N} ; W_{2}^{1}\left(\Omega_{N}\right)\right\| \leqslant c_{\delta} \varepsilon^{(1-2 \delta) / 2},
$$

где $R^{N}=v^{N}-U^{N}+Y$. Согласно построению поля $Y$ (см. п. 4) и благодаря (3.19) $R^{N}$ удовлетворяет условиям

$$
R^{N}(x)=0, \quad x \in \Gamma_{\mathrm{D}} ; \quad R_{\nu}^{N}(x), \quad x \in \Gamma_{N} .
$$

Кроме того,

$$
\begin{aligned}
L\left(\nabla_{x}\right) R^{N}(x) & =f(x)-L\left(\nabla_{x}\right) U^{N}(x)+L\left(\nabla_{x}\right) Y(x), & & x \in \Omega_{N}, \\
\sigma_{\nu \tau}\left(R^{N} ; x\right) & =-\sigma_{\nu \tau}\left(U^{N} ; x\right)+\sigma_{\nu \tau}(Y ; x), & & x \in \Gamma_{N} .
\end{aligned}
$$

Умножим скалярно уравнение (3.43) на $R^{N}$ и проинтегрируем по частям при учете краевых условий $(3.42),(3.44)$. Получим равенство

$$
\begin{aligned}
2 \mathscr{E}\left(R^{N} ; \Omega_{N}\right)= & \int_{\Omega_{N}} R^{N} \cdot\left(f-L U^{N}\right) d x-\int_{\Gamma_{N}} R_{\tau}^{N} \sigma_{\nu \tau}\left(U^{N}\right) d s_{x} \\
& +\int_{\Omega_{N}} R^{N} \cdot L Y d x+\int_{\Gamma_{N}} R_{\tau}^{N} \sigma_{\nu \tau}\left(U^{N}\right) d s_{x}=I_{1}-I_{2}+I_{3}+I_{4} .
\end{aligned}
$$

Оценим интегралы $I_{i}$. В соответствии с (3.39) и (3.44) имеем

$$
\begin{aligned}
& \left|I_{1}\right| \leqslant c \varepsilon^{1 / 2}|\ln \varepsilon|^{3}\left\|\mathscr{R} R^{N} ; L_{2}\left(\Omega_{N}\right)\right\|, \\
& \left|I_{2}\right| \leqslant c \varepsilon^{1 / 2}|\ln \varepsilon| \times\left\|\mathscr{R} R^{N} ; L_{2}\left(\Omega_{N}\right)\right\| .
\end{aligned}
$$

Пользуясь (3.26), заключаем, что

$$
\left\|L Y ; V_{1}^{0}\left(\Omega_{N} ; \mathscr{P}\right)\right\| \leqslant c\left\|Y ; V_{1}^{2}\left(\Omega_{N} ; \mathscr{P}\right)\right\| \leqslant c_{\delta} \varepsilon^{(3-4 \delta) / 2} .
$$

Так как поле $Y$, а значит, и поле $L Y$ равны нулю в $c \varepsilon^{2}$-окрестностях точек $P^{1}, \ldots, P^{N}$ (см. текст после (3.24)), то

$$
\begin{aligned}
& \left\|\mathscr{R}^{-1} L Y ; L_{2}\left(\Omega_{N}\right)\right\| \\
& \quad \leqslant c \sup \left\{\mathscr{R}(x)^{-1} \operatorname{dist}(x, \mathscr{P})^{-1} \mid x \in \operatorname{supp} L Y\right\} \times\left\|L Y ; V_{1}^{0}\left(\Omega_{N} ; \mathscr{P}\right)\right\| \\
& \quad \leqslant c \varepsilon^{-1}|\ln \varepsilon| \times\left\|L Y ; V_{1}^{0}\left(\Omega_{N} ; \mathscr{P}\right)\right\| \leqslant c_{\delta} \varepsilon^{(1-6 \delta) / 2}
\end{aligned}
$$

и

$$
\left|I_{3}\right| \leqslant c_{\delta} \varepsilon^{(1-6 \delta) / 2}\left\|\mathscr{R} R^{N} ; L_{2}\left(\Omega_{N}\right)\right\| .
$$

Поскольку согласно свойствам пространств Кондратьева

$$
\begin{aligned}
\left\|\sigma_{\nu \tau}(Y) ; L_{2}\left(\Gamma_{N}\right)\right\| & \leqslant c\left\|Y ; V_{0}^{1}\left(\Gamma_{N} ; \mathscr{P}\right)\right\| \\
& \leqslant c\left\|Y ; V_{1 / 2}^{3 / 2}\left(\Gamma_{N} ; \mathscr{P}\right)\right\| \leqslant c\left\|Y ; V_{1 / 2}^{2}\left(\Omega_{N} ; \mathscr{P}\right)\right\| \\
& \leqslant c \sup \left\{\operatorname{dist}(x, \mathscr{P})^{-1 / 2} \mid x \in \operatorname{supp} Y\right\} \times\left\|Y ; V_{1}^{2}\left(\Omega_{N}\right)\right\| \\
& \leqslant c \varepsilon^{-1}\left\|Y ; V_{1}^{2}\left(\Omega_{N}\right)\right\| \leqslant c \varepsilon^{(1-4 \delta) / 2},
\end{aligned}
$$


то аналогично предыдущему

$$
\left|I_{4}\right| \leqslant c\left\|\sigma_{\nu \tau}(Y) ; L_{2}\left(\Gamma_{N}\right)\right\| \times\left\|R^{N} ; L_{2}\left(\Gamma_{N}\right)\right\| \leqslant c \varepsilon^{(1-4 \delta) / 2}\left\|R^{N} ; L_{2}\left(\Gamma_{N}\right)\right\| .
$$

Указанные неравенства для $\left|I_{i}\right|$ доставляют мажоранту правой части (3.45). В силу предложений 3.1-3.3

$$
\mathscr{E}\left(R^{N} ; \Omega_{N}\right) \geqslant c\left\|R^{N} ; W_{2}^{1}\left(\Omega_{N}\right)\right\| \times\left\{\left\|\mathscr{R} R^{N} ; L_{2}\left(\Omega_{N}\right)\right\|+\left\|R^{N} ; L_{2}\left(\Gamma_{N}\right)\right\|\right\}
$$

Сокрашая в полученном из (3.45) неравенстве слева и справа нормы, выделенные в (3.46) фигурными скобками, выводим соотношение (3.41) (не должен смущать тот факт, что вычитаемые в показателях степеней различаются: число $\delta>0-$ произвольно).

8. Теоремы о сходимости. При малом $\varepsilon$ вне любой фиксированной окрестности $\mathscr{V}$ контура $\Gamma_{0}$ срезающие функции $X$ и $\chi^{j}$ равны соответственно 1 и 0 , слагаемое $\varepsilon w$ типа пограничного слоя мало (например, благодаря представлению (3.36)). Поэтому из теоремы 3.6 и формулы (3.3) для глобального приближения вытекает следуюшее утверждение.

Tеорема 3.7. Решение $v^{N}$ задачи (1.5)-(1.7) сходится при $N \rightarrow \infty$ по метрике пространства $W_{2}^{1}(\Omega \backslash \mathscr{V}) \kappa$ решению $v$ задачи (1.8)-(1.11); здесь $\mathscr{V}$ произвольная окрестность контура $\Gamma_{0}$.

\section{§4. Другие типы краевых условий}

1. Краевые условия Дирихле и Неймана. Дополним уравнения (1.5), (1.6) условиями Дирихле

$$
v^{N}(x)=0, \quad x \in \Gamma_{N}
$$

(граница $\Gamma_{N}$ жестко защемлена), или условиями Неймана

$$
\sigma_{\nu \nu}\left(v^{N} ; x\right)=\sigma_{\nu \tau}\left(v^{N} ; x\right)=0, \quad x \in \Gamma_{N}
$$

(граница $\Gamma_{N}$ свободна от напряжений). Краевые условия (4.1), (4.2) устойчивы относительно предельного перехода $N \rightarrow \infty$ в том смысле, что в задаче для $v \mathrm{k}$ уравнениям $(1.10),(1.11)$ присоединяются соответствующие краевые условия

$$
v(x)=0, \quad x \in \Gamma_{0},
$$

или

$$
\sigma_{n s}(v ; x)=\sigma_{n n}(v ; x)=0, \quad x \in \Gamma_{0} .
$$

Иными словами, парадокс, обсуждаемьй в статье, отсутствует. Этот факт проверяется, например, при помоши подхода, развитого в [15]. Разумеется, то же самое можно показать, построив асимптотическое представление решения задачи $(1.5),(1.6),(4.1)$ или (1.5), (1.6), (4.2). Заметим, что все неприятности с предельным переходом $N \rightarrow \infty$ вызваны наличием степенных решений (2.16) с малым показателем $\Lambda$ (см. (2.17)). Для задачи Дирихле таких решений вообще нет $(|\Lambda|>1 / 2$ для любого угла; см. [28]). Это обстоятельство изымает из асимптотической схемы переразложения (2.19) и поправки (3.2) вблизи угловых точек. Таким образом, вывод предельных условий (4.3) становится совсем простым. 
Если вектор (2.16) - степенное решение задачи Неймана в каком-либо угле и $|\Lambda|<1 / 2$, то $\Lambda=0$ и (2.16) - линейная комбинация жестких поступательных смещений $r^{0} \Phi^{10}(\varphi), r^{0} \Phi^{20}(\varphi), r^{0} \Phi^{11}(\varphi, \ln r), r^{0} \Phi^{21}(\varphi, \ln r)$ и полей, порожденных сосредоточенными силами (см. [28] и ср. с (2.18)). Нетрудно убедиться в том, что при построении асимптотики $\Phi^{i 1}$ не появляются, а жесткие смещения $\Phi^{i 0}$, будучи гладкими функциями, не нуждаются в поправках типа (3.2). Опять-таки, повторяя (с упомянутыми упрощениями) рассуждения из предыдущих параграфов, приходим к краевым условиям (4.4).

2. Краевые условия (1.14). Обратимся к задаче (1.5), (1.6), (1.14) (на контуре фиксируются касательные смещения и нормальные напряжения). Как и основная задача (1.5)-(1.7), предел решения $v^{N}$ не удовлетворяет ожидаемому граничному условию

$$
v_{s}(x)=0, \quad \sigma_{n n}(v ; x)=0, \quad x \in \Gamma_{0} .
$$

Вместо него появляется краевое условие

$$
\begin{gathered}
v_{s}(x)=0, \\
\sigma_{n n}(v ; x)= \pm \frac{1}{2} v_{n}(x) \mathbb{k}(s)\left[\lambda\left(1-\frac{1}{\varkappa}\right)(1 \mp 1)+2 \mu\right. \\
\left.+\mu\left(1-\frac{1}{\varkappa} \pm\left(1+\frac{1}{\varkappa}\right)\right)\right], \quad x \in \Gamma_{0} .
\end{gathered}
$$

Этот факт устанавливается прямым повторением рассуждений из $\S 2,3$, и мы ограничимся перечислением тех формул и утверждений, которые нужно модифицировать.

В качестве главного члена разложения задачи (1.5), (1.6), (1.14) выбирается гладкая в $\bar{\Omega}$ функция $v$, удовлетворяющая условиям (1.5), (1.6). Соответствуюший пограничньй слой $\varepsilon w^{0}(x)$ должен компенсировать главные части невязок, оставляемые $v$ на $\Gamma_{N}$,

$$
\begin{gathered}
w_{1}^{0}\left(s, \xi_{1}, 0\right)=\mathbb{k}(s)\left\{V_{n}^{0}(s)\left(\xi_{1}-\frac{1}{2} \operatorname{sgn}\left(\xi_{1}\right)\right)-\frac{1}{2} V_{s}^{1}(s)\left[\left(\left|\xi_{1}\right|-\frac{1}{2}\right)^{2}-\frac{1}{4}\right]\right\}, \\
2 \mu \frac{\partial w_{2}^{0}}{\partial \xi_{2}}+\lambda\left(\frac{\partial w_{2}^{0}}{\partial \xi_{2}}+\frac{\partial w_{1}^{0}}{\partial \xi_{1}}\right)=-\Sigma_{n n}(s) .
\end{gathered}
$$

Специальные решения в угле с раствором $\alpha$ принимают в полярных координатах вид (2.7), а угловые части задаются равенствами

$$
\begin{aligned}
\Phi_{r}^{+}(\varphi) & =\cos \frac{\pi \varphi}{2 \alpha}, & \Phi_{\varphi}^{+}(\varphi) & =-\sin \frac{\pi \varphi}{2 \alpha} ; \\
\Phi_{r}^{-}(\varphi) & =\left(\varkappa-1+\frac{\pi}{2 \alpha}\right) \cos \frac{\pi \varphi}{2 \alpha}, & \Phi_{\varphi}^{-}(\varphi) & =-\left(\varkappa+1-\frac{\pi}{2 \alpha}\right) \sin \frac{\pi \varphi}{2 \alpha} .
\end{aligned}
$$

Для полуплоскости $(\alpha=\pi / 2)$ имеются решения (2.18), причем

$$
\begin{aligned}
\Phi_{r}^{0}(\varphi) & =\cos \varphi, & \Phi_{\varphi}^{0}(\varphi) & =-\sin \varphi ; \\
\Phi_{r}^{1}(\varphi, \ln r) & =\ln r \cos \varphi-(2 \varkappa)^{-1} \cos \varphi, & \Phi_{\varphi}^{1}(\varphi, \ln r) & =-\ln r \sin \varphi-(2 \varkappa)^{-1} \sin \varphi .
\end{aligned}
$$


Разложение (2.19) заменяется следуюшим:

$$
r^{\mp 1 \pm \pi /(2 \alpha)} \Phi^{ \pm}(\varphi)=\Phi^{0}(\varphi)+2 \pi^{-1} \delta\left[\Phi^{1}(\varphi, \ln r) \pm \Psi(\varphi)\right]+O\left(\delta^{2}|\ln r|^{2}\right) ;
$$

здесь

$$
\begin{aligned}
\Psi_{r}(\varphi) & =-\varphi \sin \varphi+(2 \varkappa)^{-1} \cos \varphi \\
\Psi_{\varphi}(\varphi) & =-\varphi \cos \varphi+(2 \varkappa)^{-1} \sin \varphi
\end{aligned}
$$

Теперь асимптотическое условие (2.24) выглядит так:

$$
\begin{aligned}
w(s, \xi)= & \pi^{-1} \mathbb{k}(s) V_{n}^{0}(s)\left[ \pm \Phi^{1}(\varphi, \ln r)+\Psi(\varphi)\right] \\
& +C(s) \Phi^{0}(\varphi)+O(r|\ln r|), \quad r=|\xi| \rightarrow 0 .
\end{aligned}
$$

Наконец, простая переформулировка теоремы 2.1 (со ссылками на общие результаты [27]) и соблюдение условий отсутствия роста решения типа пограничного слоя приводят к предельному условию (4.5).

\section{Список литературы}

1. Гольденвейзер A.Л. Построение приближенной теории изгиба пластинки методом асимптотического интегрирования уравнений теории упругости // Прикладная матем. и механика. 1962. Т. 27. №6. С. 1057-1074.

2. Михлин C. Г. Вариационные методы в математической физике. М.: ГИТТЛ, 1957.

3. Сапонджкян О. М. Изгиб тонких упругих плит. Ереван: Айастан, 1975.

4. Бабушка И. Устойчивость области определения по отношению к возмущению границы основных задач теории дифференциальных уравнений в частных производных, главным образом в связи с теорией упругости. 1 // Чехословацкий матем. журн. 1961. Т. II. № 1. C. 76-105; 2 // Чехословацкий матем. журн. 1961. Т. II. № 2. C. 165-203.

5. Фикера $Г$. Теоремы существования в теории упругости. М.: Мир, 1974.

6. Ciarlet P. G. Plates junctions in elastic microstructures. Paris-N.Y.: MASSON, 1980.

7. Сапонджяян О. М. Изгиб свободно опертой полигональной плиты // Изв. АН АрмССР. 1952. T. 5. № 2. C. 29-46.

8. Мазья B. Г., Назаров $C$. А. Парадоксы предельного перехода в решениях краевых задач при аппроксимации гладких контуров многоугольными // Изв. АН СССР. Сер. матем. 1986. T. 50. №6. C. 1156-1177.

9. Rajaiah K., Rao A.K. Effect of Boundary Conditions Description on Convergence of Solution in a Boundary-Value Problem // J. of Computational Physics. 1968. V. 3. № 2. P. 190-201.

10. Hanuska A. Zu den Theorien der Plattenbiegung // Beton-und-Stahlbetonbau. 1969. V. 9. P. 214-217.

11. Rieder G. Eingrenzungen in der Elastizitats- und Potential-theorie // ZAMM. 1972. Bd. 52. № 10. S. 340-347.

12. Murray N.W. The Polygone-Circle Paradox and Convergence in Thin Plate Theory // Proceedings of the Cambridge Philosophical Society. 1973. V. 73. P. 279-283.

13. Rieder G. On the Plate Paradox of Sapondzhyan and Babuska // Mech. Res. Comm. 1974. V. 1. P. 51-53.

14. Rajaiah K., Rao A. K. On the Polygone-Circle Paradox // J. of Applied Mechanics. 1981. V. 48. P. $195-196$.

15. Babuska I., Pitkaranta J. The plate paradox for hard and soft simple support // SIAM J. Math. Anal. 1990. V. 21. № 3. P. 551-576.

16. Reissner E. On the bending of elastic plates // Quart. Appl. Math. 1947. V. 5. № 1.

17. Тимошенко C.П., Войновский-Кригер C. Пластинки и оболочки. М.: Физматгиз, 1963. 
18. Шойхет Б. А. Одно энергетическое тождество в физически нелинейной теории упругости и оценки погрешностей уравнений плит // Прикл. матем. и механика. 1976. Т. 40. № 2. C. $317-326$.

19. Зорин И. С., Назаров С.А. Краевой эффект при изгибе тонкой трехмерной пластины // Прикл. матем. и механика. 1989. Т. 53. № 4. С. 642-650.

20. Bensoussan A., Lions J.-L., Papanicolaou G. Asymptotic analysis for periodic structures. Amsterdam: North'Holland Publ. Co., 1978.

21. Бахвалов Н. С., Панасенко Г. П. Осреднение процессов в периодических средах. М.: Наука, 1984.

22. Санчес-Паленсия Э. Неоднородные среды и теория колебаний. М.: Мир, 1984.

23. Олейник О.А., Иосифьян Г.А., Шамаев А. С. Математические задачи теории сильно неоднородных сред. М.: Изд-во МГУ, 1990.

24. Жиков В.В., Козлов С. М., Олейник О. А. Усреднение дифференциальных операторов. М.: Наука, 1993.

25. Кондратьев B. A. Краевые задачи для эллиптических уравнений в областях с коническими или угловыми точками // Тр. Моск. матем. об-ва. 1967. Т. 16. С. 209-298.

26. Мазья В.Г., Пламеневский Б. А. О коэффициентах в асимптотике решений эллиптических краевых задач в областях с коническими точками // Math. Nachr. 1977. Bd. 76. S. 29-60.

27. Назаров С. А., Пламеневский Б. А. Эллиптические задачи в областях с кусочно гладкой границей. М.: Наука, 1991.

28. Партон В.З., Перлин П. И. Методы математической теории упругости. М.: Наука, 1981.

29. Олейник О.А., Иосифьян Г.А. О поведении на бесконечности решений уравнений второго порядка в области с некомпактной границей // Матем. сб. 1980. Т. 112. № 4 . C. $588-610$.

30. Oleinik O.A., Yosifian G. A. On the asymptotic behavior at infinity of solutions in linear elasticity // Arch. Rat. Mech. Anal. 1982. V. 78. № 1. P. 29-53.

31. Agmon S., Douglis A., Nirenberg L. Estimates near the boundary for solutions of elliptic differential equations satisfying general boundary conditions // Comm. Pure Appl. Math. 1964. V. 17. P. 35-92.

32. Берс Л., Джсон Ф., Шехтер М. Уравнения с частными производными. М.: Мир, 1966.

33. Fridrichs K. O. On the boundary value problems of the theorie of elasticity and Korn's inequality // Ann. Math. 1947. V. 48. P. 447-471.

34. Дюво Г., Лионс ЖК.-Л. Неравенства в механике и физике. М.: Наука, 1980.

35. Кондратьев B.A., Олейник O.A. Краевые задачи для системы теории упругости в неограниченных областях. Неравенство Корна // УМН. 1988. Т. 43. № 5. С. 55-98.

36. Кондратьев B.A., Олейник О.А. О зависимости констант в неравенстве Корна от параметра, характеризующего геометрию области // УМН. 1989. Т. 44. № 6. С. 157-158.

37. Kondratiev V.A., Oleinik O.A. Hardy's and Korn's type inequalities and their applications // Rendiconti di Matematica. Ser. VII. Roma. 1990. V. 10. P. 641-666.

38. Лады женская O. А. Краевые задачи в математической физике. М.: Наука, 1973.

39. Mazja W.G., Nazarov S.A., Plamenevski B.A. Asymptotische Theorie elliptischer Randwertaufgaben in singular gestorten Gebieten. Berlin: Akademie-Verlag, 1991. Bd. 1, 2. 